University of Tennessee Health Science Center UTHSC Digital Commons

\title{
$5-2016$
}

\section{Novel Oncogenic Drivers in Pediatric Gliomagenesis}

Alexander K. Diaz

University of Tennessee Health Science Center

Follow this and additional works at: https://dc.uthsc.edu/dissertations

Part of the Genetic Processes Commons, and the Neoplasms Commons

\section{Recommended Citation}

Diaz, Alexander K. (http://orcid.org/0000-0002-5558-6825), "Novel Oncogenic Drivers in Pediatric Gliomagenesis" (2016). Theses and Dissertations (ETD). Paper 373. http://dx.doi.org/10.21007/ etd.cghs.2016.0396.

This Dissertation is brought to you for free and open access by the College of Graduate Health Sciences at UTHSC Digital Commons. It has been accepted for inclusion in Theses and Dissertations (ETD) by an authorized administrator of UTHSC Digital Commons. For more information, please contact jwelch30@uthsc.edu. 


\title{
Novel Oncogenic Drivers in Pediatric Gliomagenesis
}

\begin{abstract}
Pediatric high-grade gliomas (pHGGs), with a two-year survival rate of less than $20 \%$, are some of the most aggressive human cancers. This dissertation begins with our analysis of $127 \mathrm{pHGGs,} \mathrm{including}$ brainstem (BS) and non-brainstem (NBS) tumors, from 118 patients using next-generation sequencing technologies. Nearly one-third of BS-HGGs, also known as diffuse intrinsic pontine gliomas (DIPGs), harbored somatic heterozygous missense mutations in $A C V R 1$, coding for a receptor serine-threonine kinase involved in bone morphogenetic protein (BMP) signaling. These alterations led to gain-of-function as evidenced by increased phosphorylation of downstream targets in primary astrocytes and zebrafish embryo ventralization. Whole-genome sequencing and RNASeq revealed that nearly half of our cohort contained structural variants. We identified recurrent gene fusions preserving the kinase domain of the neurotrophin family of receptor tyrosine kinases (NTRK) including three novel fusions and two fusions previously described in other tumor types. NTRK fusion genes were identified in $40 \%$ of infant ( $<3$ years of $>$ age) NBS-HGGs, and $7 \%$ of pHGG overall. We also found that infants have significantly reduced mutation burdens when compared to $\mathrm{pHGGs}$ in older children, suggesting a small number of oncogenic mutations are required in infant tumors. These findings, coupled with the observation that infants have a better prognosis than non-infants, make infant NBS tumors a distinct subgroup of pHGG. NTRK gene fusions also occur in pediatric low-grade glioma ( $\mathrm{pLGG)}$ ) and adult glioblastoma but are not as enriched as they are in infant NBS-HGG, and adult glioblastoma and non-infant pHGGs exhibit higher mutation rates than infant tumors. NTRK fusion genes are therefore gliomagenic drivers throughout various development settings; yet it appears as if gliomas driven by the same oncogenic lesion can vary in tumor phenotype as a function of contextual differences. With this in mind, we used genetically engineered mice with a NTRK gene fusion knock-in allele to generate HGG in vivo. Given that tumor is evident by early postnatal life (P5), this is, to our knowledge, the first report of a bona fide spontaneous pHGG model. The second part of this dissertation is the characterization of these tumors.
\end{abstract}

\section{Document Type}

Dissertation

Degree Name

Doctor of Philosophy (PhD)

Program

Biomedical Sciences

Research Advisor

Suzanne J. Baker, Ph.D.

Keywords

ACVR1, cancer, gene fusions, glioma, NTRK

\section{Subject Categories}

Diseases | Genetic Processes | Medical Sciences | Medicine and Health Sciences | Neoplasms 


\title{
Novel Oncogenic Drivers in Pediatric Gliomagenesis
}

\author{
A Dissertation \\ Presented for \\ The Graduate Studies Council \\ The University of Tennessee \\ Health Science Center \\ In Partial Fulfillment \\ Of the Requirements for the Degree \\ Doctor of Philosophy \\ From The University of Tennessee
}

By

Alexander K. Diaz

May 2016 
Portions of Chapter 1 (C) 2014 by Elsevier. Chapter 2 (C) 2014 by Nature Publishing Group. All other material $\odot 2016$ by Alexander K. Diaz. All rights reserved. 


\section{DEDICATION}

To the people in my life that make each day the gift it is. 


\section{ACKNOWLEDGEMENTS}

This work would not have been possible without Dr. Suzanne J. Baker. It has been nothing short of an honor to both know her and work with her. I walk away from this experience a better person and for that she deserves much of the credit. It is because of her that I will be able to reassure my future patients and their families that things will get better.

In these last few years the lab has become my family (although a much quieter version than what I'm normally accustomed to). And like any family, they have carried me through difficult times in getting me to this point. I will miss them dearly.

I have had the privilege of working with various clinical colleagues along the way: Drs. David Ellision and Brent Orr in Pathology, Dr. Patay in Diagnostic Imaging, and Drs. Justin Baker and Amar Gajjar in Oncology.

Countless individuals have worked tirelessly in helping me with experiments and to them I am sincerely grateful. Specifically I'd like to thank Dr. Chris Calabrese, Brittney Gordon, Shantel Brown, and Melissa Johnson. This work would not have been possible without the heroic efforts of Drs. Jinghui Zhang, Gang Wu, and Chunxu Qu.

Most importantly, I humbly thank St. Jude patients and families. Their suffering is something no one should ever know. They place a tremendous amount of faith in us, and it is my hope that my work is in some way worthy of that trust. 


\begin{abstract}
Pediatric high-grade gliomas (pHGGs), with a two-year survival rate of less than $20 \%$, are some of the most aggressive human cancers. This dissertation begins with our analysis of 127 pHGGs, including brainstem (BS) and non-brainstem (NBS) tumors, from 118 patients using next-generation sequencing technologies. Nearly one-third of BS-HGGs, also known as diffuse intrinsic pontine gliomas (DIPGs), harbored somatic heterozygous missense mutations in $A C V R$ 1, coding for a receptor serine-threonine kinase involved in bone morphogenetic protein (BMP) signaling. These alterations led to gain-of-function as evidenced by increased phosphorylation of downstream targets in primary astrocytes and zebrafish embryo ventralization. Whole-genome sequencing and RNASeq revealed that nearly half of our cohort contained structural variants. We identified recurrent gene fusions preserving the kinase domain of the neurotrophin family of receptor tyrosine kinases (NTRK) including three novel fusions and two fusions previously described in other tumor types. NTRK fusion genes were identified in $40 \%$ of infant ( $<3$ years of age) NBS-HGGs, and 7\% of pHGG overall. We also found that infants have significantly reduced mutation burdens when compared to pHGGs in older children, suggesting a small number of oncogenic mutations are required in infant tumors. These findings, coupled with the observation that infants have a better prognosis than non-infants, make infant NBS tumors a distinct subgroup of pHGG. NTRK gene fusions also occur in pediatric low-grade glioma (pLGG) and adult glioblastoma but are not as enriched as they are in infant NBS-HGG, and adult glioblastoma and non-infant pHGGs exhibit higher mutation rates than infant tumors. NTRK fusion genes are therefore gliomagenic drivers throughout various development settings; yet it appears as if gliomas driven by the same oncogenic lesion can vary in tumor phenotype as a function of contextual differences. With this in mind, we used genetically engineered mice with a NTRK gene fusion knock-in allele to generate HGG in vivo. Given that tumor is evident by early postnatal life (P5), this is, to our knowledge, the first report of a bona fide spontaneous pHGG model. The second part of this dissertation is the characterization of these tumors.
\end{abstract}




\section{TABLE OF CONTENTS}

CHAPTER 1. INTRODUCTION .................................................................................

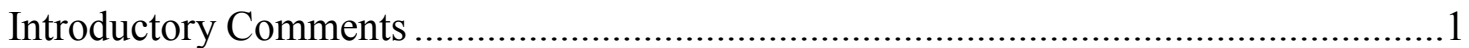

High-Grade Gliomas .......................................................................................2

The Genetics of Adult and Pediatric High-Grade Glioma...........................................4

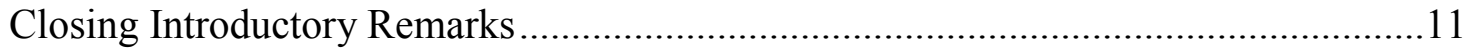

CHAPTER 2. THE GENOMIC LANDSCAPE OF DIFFUSE INTRINSIC PONTINE GLIOMA AND PEDIATRIC NON-BRAINSTEM HIGH-GRADE

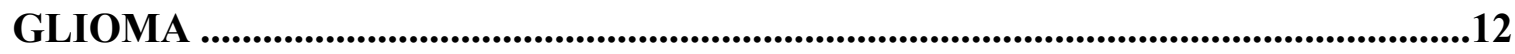

CHAPTER 3. FUSIONS INVOLVING THE NEUROTROPHIN FAMILY OF RECEPTOR TYROSINE KINASES IN GLIOMAGENESIS .......................................25

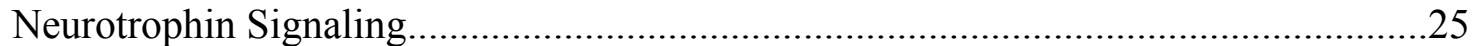

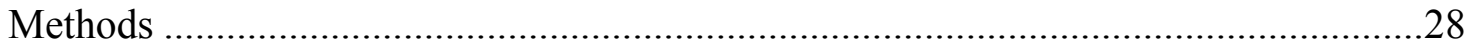

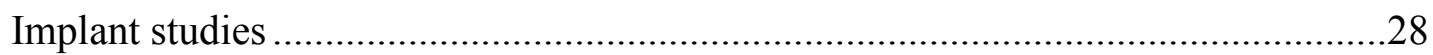

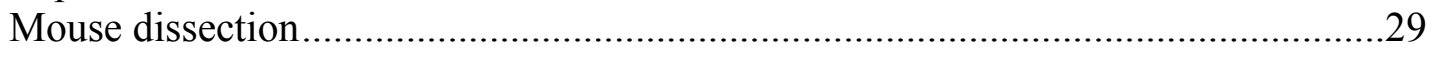

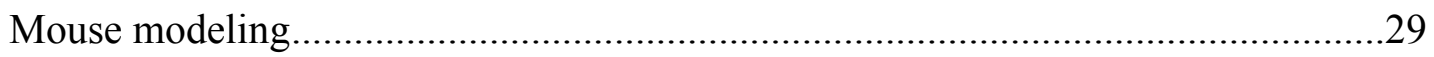

Immunohistochemistry/Immunofluorescence ....................................................29

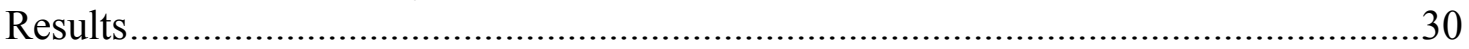

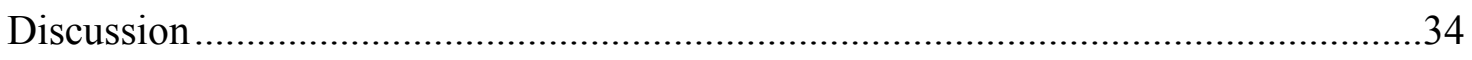

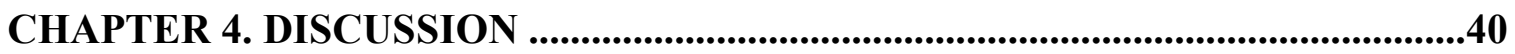

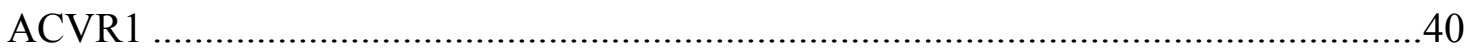

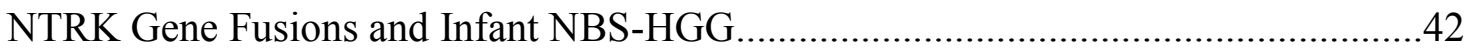

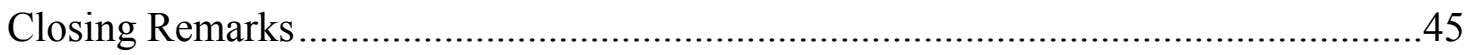

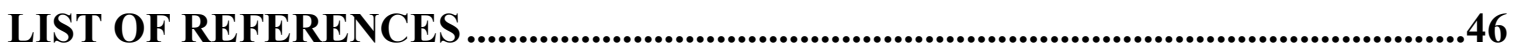

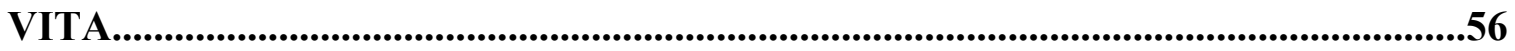




\section{LIST OF FIGURES}

Figure 1-1. The p53, RB, and RTK-Ras-PI3K pathways are dysregulated in both adult and pediatric HGG.

Figure 1-2. Hot-spot histone mutations occur in nearly $80 \%$ of DIPGs and $\sim 35 \%$ of NBS-HGGs.

Figure 1-3. Histone mutations are strongly associated with anatomical location. 10

Figure 2-1. Recurrent genetic alterations in pediatric HGG. 14

Figure 2-2. ACVR1 mutations in DIPG activate BMP signaling. 15

Figure 2-3. Three structural variants generate oncogenic chimeric NTRK fusion proteins.

Figure 2-4. Pediatric HGG alterations in histone modifiers or chromatin regulators. ....19

Figure 2-5. Circos plots showing the range of structural alterations in pediatric HGG.

Figure 2-6. Outlier analysis of the tier1 mutation rate in all pediatric HGGs. .23

Figure 3-1. The NTRK signaling pathway.

Figure 3-2. Astrocytes expressing ETV6-NTRK3 generate HGG when implanted into recipient mice.

Figure 3-3. Expression of EN3 confers a proliferative advantage in vitro.

Figure 3-4. Kaplan-Meier curve of five cohorts: $N C r e ; E N 3 ; \operatorname{Trp} 53^{--}$(red), NCre;EN3;Trp53+/ (black), NCre;EN3 (purple), NCre;Trp 53-- (blue), and $N C r e ; T r p 53^{+/-}$(green).

Figure 3-5. NCre;EN3;Trp $53^{-/-}$mice generate HGG.

Figure 3-6. Tumors from NCre;EN3;Trp $53^{-/-}$mice show activation of PI3K and MAPK.

Figure 3-7. NCre;EN3;Trp53-/- mice have high-grade disease by P5.

Figure 3-8. Tumorspheres from NCre;EN3;Trp $53^{-/-}$mice generate HGGs when implantated into recipient mice. 


\section{LIST OF ABBREVIATIONS}

aHGG

ALL

BDNF

BMP

CNS

DAG

DIPG

EGFR

EGFRvIII

ER

FOP

GFAP

GS

HGG

IHC

IP3

LGG

$\mathrm{LOH}$

MAPK

MGNT

MLL

MSCV
Adult high-grade glioma

Acute lymphoblastic leukemia

Brain-derived neurotrophic factor

Bone morphogenetic protein

Central nervous system

Diacylglycerol

Diffuse intrinsic pontine glioma

Epidermal growth factor receptor

Epidermal growth factor receptor, variant III

Endoplasmic reticulum

Fibrodysplasia ossificans progressive

Glial fibrillary acidic protein

Glycine-serine

High-grade glioma

Immunohistochemistry

Inositol trisphosphate

Low-grade glioma

Loss of heterozygosity

Mitogen-activated protein kinase

Malignant glioneuronal tumor

Mixed-lineage leukemia

Murine stem cell virus 


\begin{tabular}{|c|c|}
\hline NBS & Non-brainstem \\
\hline NGF & Nerve growth factor \\
\hline NSC & Neural stem cell \\
\hline NTRK & Neurotrophic tyrosine receptor kinase \\
\hline $\mathrm{OPC}$ & Oligodendrocyte precursor cell \\
\hline PDGFR $\alpha$ & Platelet-derived growth factor receptor, alpha \\
\hline pHGG & Pediatric high-grade glioma \\
\hline PIP2 & Phosphatidylinositol 4,5-bisphosphate \\
\hline PIP3 & Phosphatidylinositol $(3,4,5)$-trisphosphate \\
\hline PI3K & Phosphotidylinositide 3-kinase \\
\hline PKC & Protein kinase $\mathrm{C}$ \\
\hline $\mathrm{PLC} \gamma$ & Phospholipase C, gamma \\
\hline pNBS-HGG & Pediatric non-brainstem high-grade glioma \\
\hline PTEN & Phosphatase and tensin homolog \\
\hline $\mathrm{RB}$ & Retinoblastoma \\
\hline RTK & Receptor tyrosine kinase \\
\hline SNV & Single-nucleotide variation \\
\hline SVZ & Subventricular zone \\
\hline TGF $\beta$ & Transforming growth factor beta \\
\hline WT & Wild-type \\
\hline WHO & World Health Organization \\
\hline
\end{tabular}




\section{CHAPTER 1. INTRODUCTION}

\section{Introductory Comments}

Cancer is unequivocally a genetic disease. That fact is so fundamental to our understanding that we oftentimes take it for granted. Indeed, it is difficult for us to even fathom a paradigm that predates our own. However, the history of cancer, or rather its written history, stretches as far back as 3000 BC with Ancient Egyptian descriptions of breast tumors. And since then, theories of cancer etiology have been just as varied as the cancer themselves; from explanations such as gods and humoral imbalance to more recent hypotheses including lymph and blastema theory[1]. The isolation of DNA in 1869[2] did not immediately seem relevant to the study of cancer, and it would take until the mid- $20^{\text {th }}$ century for the inception of oncogene theory[3]. Since that time, cancer biology has become predicated upon genetic alterations leading to gain-and-loss-offunction of proteins involved in every cellular function imaginable, from signaling cascades promoting cell survival and proliferation to DNA damage repair to epigenetic regulation. The combination of events leading to neoplasia are varied, perhaps innumerable. Yet regardless of its complexity, cancer is a disease of a singular process: perturbation conferring a selective advantage.

The role of genetic alterations in cancer, both inherited and acquired, is an obvious one. What has become evident more recently is the idea that cancers arising in adults are different from those in children. There are common cases for adult oncologists, such as lung cancer, that are rare for pediatric physicians and vice versa[4]. True as that may be, for cancers that occur throughout life the histopathology is indistinguishable between adults and children. This in turn led to the thinking that similar histology was the result of similar underlying genetics; resulting in the thinking that children are "little adults." Fast forward to the $21^{\text {st }}$ century. We can comfortably assert, informed by decades of genetic and molecular analyses, that such thinking is decidedly wrong. That is not to say there is no overlap whatsoever. Adult and pediatric cancers are indeed related, but they are also distinct. One issue that remains unresolved is the idea that pediatric cancers arise from an underlying genetic susceptibility. Many believe that the only way children can acquire the necessary hits for tumor formation, given their reduced latency, is through germline mutations leading to cancer predisposition syndromes. After sequencing thousands of tumor/matched normal pairs, we are still uncertain whether this is indeed the case or not. Only a minority of children who develop cancer carry germline mutations in known susceptibility genes[5]. However, our understanding of protein coding sequences is far from comprehensive and that of the remaining $98 \%$ of the noncoding genome is even less; so the functional consequence of the ever-expanding catalog of germline variants identified in pediatric cancer patients remains to be seen[6]. What

* Reprinted with permission. Diaz, A.K. and S.J. Baker, The genetic signatures of pediatric high-grade glioma: no longer a one-act play. Semin Radiat Oncol, 2014. 24(4): p. 240-7. 
this data has also revealed is that generally speaking, pediatric cancers exhibit lower mutation burdens than cancers more commonly seen in adults. Interestingly, but not altogether surprising, malignancies with known associations with environmental mutagens, such as lung and colorectal cancers, have some of the highest mutation burdens evident[7]. But it is important to keep in mind that there also exists heterogeneity within a given tumor type. For example, pediatric high-grade gliomas, an aggressive brain tumor that will be the focus of this dissertation, range from some of the quietest cancer genomes to having a hypermutator phenotype[8-11].

The Genomic Era continues onward, but it has already yielded critical insight. The differences between pediatric and adult cancers highlight the central importance developmental context plays in pathogenesis. But rather than merely serving as a backdrop, developmental context is an active participant, from determining the cell populations involved to influencing what genes are expressed. Yes, there are common pathways dysregulated in various cancer types regardless of age. And this should come as no surprise given their roles in cellular processes such as cell survival and proliferation. Yet when we look a bit more closely, we begin to see differences emerge as targeted effectors tend to differ between adults and children. Moreover, in certain instances we see involvement of a particular pathway/mechanism in one age group that does not appear implicated in the other.

The success of the Genomic Era goes without saying. We now have a much better idea of who the culprits are (genetic events) in tumor formation. Exactly how these alterations are contributing to cancer is now the imperative; because it is with this knowledge that we can then attempt to take advantage of them therapeutically.

\section{High-Grade Gliomas}

Experts predict that nearly 80,000 new cases of primary brain/central nervous system (CNS) tumors will be diagnosed in the United States in 2016; primary meaning disease originating in the brain/CNS compared to metastases. Those estimates also indicate that about one-third of these cases will be malignant[12]. Brain tumors present clinicians with unique challenges that differ from other cancers. For one, most deaths from brain tumors are the consequence of a growing mass encased within a confined space leading to increased intracranial pressure resulting in loss of brain function[13, 14]. Additionally, the blood-brain barrier, which works in keeping harmful substances outside of the CNS, works in keeping potentially efficacious chemotherapies out as well[15]. As a result, certain brain tumors remain some of the most lethal cancers.

Pathologists formally diagnose these cases by how they appear through the microscope; specifically, by cell morphology and protein expression via immunohistochemistry (IHC). If a tumor exhibits features of astrocytes, oligodendrocytes, or both, then it is referred to as a glioma. The most commonly used scheme for grading gliomas is that developed by the World Health Organization (WHO). It is a I-IV scale with grade I denoting the most benign form of disease and grade IV (also 
known as glioblastoma) being the most aggressive. Going from grade I to grade IV, tumors exhibit increased mitotic activity, nuclear atypia, and anaplasia. Gliomas graded III and IV are collectively known as high-grade gliomas (HGG) whereas grades I and II are regarded as low-grade gliomas (LGGs)[16].

Given the morphology that defines gliomas, it is logical to assert that they arise from glial cells. But specifically which one/s? In short, the issue remains unresolved. Gliogenesis is a highly complex process that involves a number of different cell types including stem and progenitor cells as well as transit-amplifying populations and of course fully differentiated astrocytes and oligodendrocytes. And when we take a look at the experimental data, a number of these possess the capacity to transform, leading to glioma formation. Glia formation in utero follows neurogenesis and continues throughout post-natal development. Multipotent neural stem cells (NSCs) divide asymmetrically yielding neuronal progenitors, astrocytic progenitors, and oligodendrocyte precursor cells (OPCs), with each giving rise to its differentiated namesake progeny. NSC populations persist even into adulthood, populating the subventricular zone (SVZ) and dentate gyrus, specifically the subgranular zone[17]. Evidence supporting NSCs as a putative cell-oforigin includes the ability of proliferative niches to generate gliomas in mice upon retroviral delivery of oncogenic mutations; whereas those same alterations failed to induce gliomagenesis outside of these niches[18-20]. Moreover, targeted depletion of cells expressing nestin (an intermediate filament and NSC marker) significantly impaired tumor growth in vivo[21]. As for the role of astrocytes in tumor initiation, numerous studies have been conducted taking genetically altered primary astrocyte cultures and implanting them into recipient mice with subsequent glioma formation[8, 22-29]. Spontaneous models have also implicated astrocytes; using an inducible expression system, researchers targeted Cre-mediated recombination in adult brains, specifically in cells expressing glial fibrillary acidic protein (GFAP). And although the marker is expressed both in astrocytes and NSCs, nearly a quarter of the gliomas that formed did so outside of the SVZ and subgranular zone, with no evidence of disease originating in those areas. Albeit indirectly, what this demonstrates is an increased capacity of NSCs to transform relative to astrocytes but with astrocytes still possessing such potential[30]. Aside from the growing experimental literature supporting OPCs as glioma initiating cells[31], a particular gene expression signature associated with HGG, known as the proneural subtype, shows extensive overlap with OPCs[32]. This, coupled with the fact that OPCs actually outnumber NSCs in the adult brain[33-35], leads many to believe that at least a portion of HGGs arise from this cell population.

Even prior to interrogation with microarrays or next-generation sequencing (NGS), it was reasonable to suspect that pediatric gliomas were different from those in adults. For one, the majority of adult gliomas are high-grade whereas in children, the most common gliomas are low-grade tumors. Also, where gliomas arise differs as a function of age: most gliomas in adults occur in the cerebral hemispheres while in children, a broader range of areas are affected. High-grade tumors of the brainstem are commonly referred to as diffuse intrinsic pontine gliomas (DIPGs). Brainstem HGGs almost exclusively occur in children, making it a uniquely pediatric disease, and roughly half of pediatric HGG cases are DIPGs[12, 36]. Another distinguishing feature between 
adult and pediatric gliomas is the frequency of malignant transformation, the process where a patient presents with a high-grade tumor that was previously diagnosed as a lower grade[37]. Lastly, the current standard of chemotherapeutic care for adult highgrade glioma, the alkylating agent temozolomide, has not been shown to improve survival in pediatric trials[38-40]. Collectively, these observations hinted at the possibility of pediatric HGG (pHGG) having a distinct genetic etiology from adult HGG (aHGG).

\section{The Genetics of Adult and Pediatric High-Grade Glioma}

A great deal of work has gone into uncovering the genetics underlying HGG. During my time as a graduate student, I published a review that was intended to be a comparative analysis between the adult and pediatric forms of the disease. Quoted text in this section is taken from that review[41].

Genetic studies of aHGG, specifically glioblastoma, revealed dysregulation in three main networks: the retinoblastoma (RB), p53, and receptor tyrosine kinase (RTK)Ras-phosphotidylinositide 3-kinase (PI3K) pathways[42-44]. "The p53 and RB pathways regulate G1 cell cycle checkpoints. Mitogenic signaling activates the cyclin D-dependent kinases CKD4 or CKD6, coupled with cyclin D family members (CCND1/2/3). This complex phosphorylates $\mathrm{pRB}$, releasing $\mathrm{E} 2 \mathrm{~F}$ and promoting transcription of genes responsible for G1-S cell cycle progression. The tumor suppressor locus CDKN2A encodes 2 different proteins through translation of 2 different reading frames, p16INK4A and p19ARF. p16INK4A inhibits the activity of the cyclin D-dependent kinases CKD4 and CKD6. Oncogenic signals, DNA damage, or induction of p19ARF induces p53, leading to cell cycle arrest, apoptosis, or senescence[45]. Mutations in the RTK-RasPI3K pathway transduce unregulated signals for cell proliferation, growth, and survival. RTK signaling begins when growth factor ligand binding leads to receptor dimerization. Upon dimerization, RTKs trans-phosphorylate one another at tyrosine residues in their cytosolic tails. p85, the regulatory subunit of PI3K, can then either directly bind to these phosphorylated tyrosine residues or connect to RTKs through adaptor molecules and Ras. PI3K comprises catalytic (p110) and regulatory (p85) subunits (Figure 1-1)[46].

As the genomic landscape of aHGG came into view, it shaped initial work into the pediatric disease. The first pHGG studies focused primarily on investigating the involvement of high-frequency recurrent events found in adult tumors. For example, epidermal growth factor receptor (EGFR) is the most commonly altered RTK in aHGG; with the corresponding gene locus undergoing amplification or intragenic deletion or both in $\sim 50 \%$ of cases[42-44]. First identified in adult glioblastoma, EGFR variant III (EGFRvIII) is the most common EGFR variant in aHGG and is formed by deletion of exons 2-7 resulting in a constitutively active kinase[47-49]. Accordingly, investigators early on sought to examine the degree of EGFR involvement in pediatric cases of HGG.

A number of studies found that EGFR alteration was less frequent in $\mathrm{pHGG}[50$ 56], although gene amplification and EGFRvIII expression were detected in some 
A.
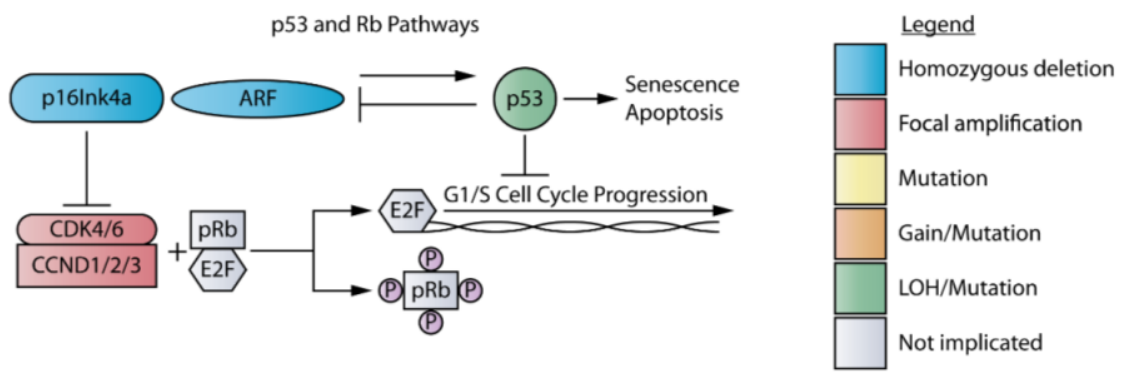

B.

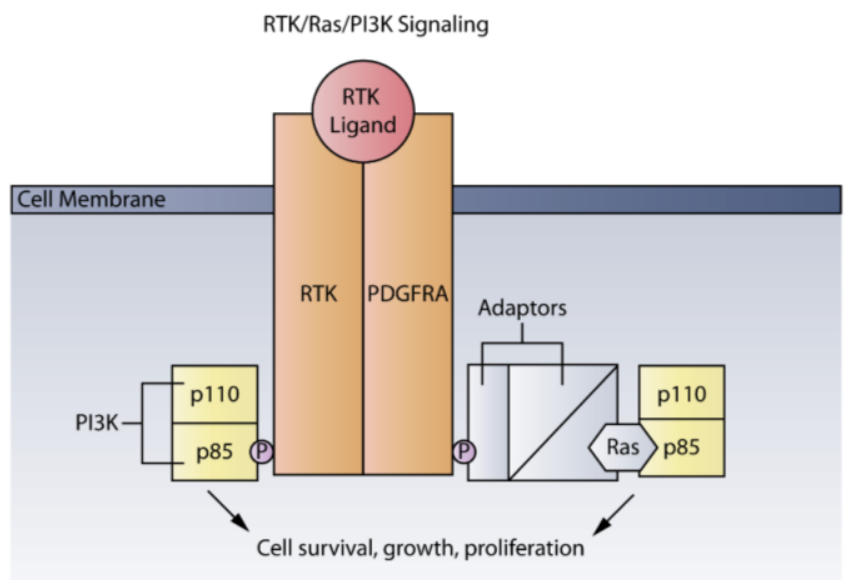

Figure 1-1. The p53, RB, and RTK-Ras-PI3K pathways are dysregulated in both adult and pediatric HGG.

(A) The p53 and RB pathways regulate G1 cell cycle checkpoints. Mitogenic signaling leads to activation of the cyclin D-dependent kinases, CKD4 or CKD6, coupled with cyclin D family members, which confer substrate specificity. CDK4/6-Cyclin D complexes, in turn, phosphorylate $\mathrm{pRB}$, facilitating E2F-mediated transcription of genes leading to G1-S cell cycle progression. Gene amplifications of $C D K 4, C D K 6$, or the 3 cyclin D family members $(C C N D 1 / 2 / 3)$ are found in $\mathrm{pHGG}$, and moreso in DIPG. The tumor suppressor locus $C D K N 2 A$ encodes 2 different proteins, p16INK4A and p19ARF. p16INK4A inhibits CDK4/6-Cyclin D complex activity. Oncogenic signals, DNA damage, or induction of p19ARF upregulates p53 expression, leading to cell cycle arrest, apoptosis, or senescence. Homozygous deletions of $C D K N 2 A$ occur almost exclusively in pediatric NBS-HGGs, whereas TP53 mutations are common regardless of age or location. (B) RTK signaling initiates when growth factors bind, leading to receptor dimerization. In $\mathrm{pHGG}$, PDGFR $\alpha$ is the most commonly targeted RTK, either by amplification or mutation or both. Upon dimerization, RTKs trans-phosphorylate one another at tyrosine residues in their cytosolic tails. These phosphorylated tyrosines can then either bind directly to $\mathrm{p} 85$, the regulatory subunit of PI3K, or bind PI3K indirectly through adaptor molecules and Ras. PI3K comprises catalytic (p110) and regulatory (p85) subunits, both of which are targeted by mutation, usually in a mutually exclusive pattern, in pHGG. $\mathrm{pRB}, \mathrm{RB}$ protein. 
pHGGs[57-60]. Through genome-wide studies, PDGFRA, which encodes plateletderived growth factor receptor alpha (PDGFR $\alpha$ ), was identified as the most commonly targeted RTK in both DIPG and pediatric non-brainstem HGG (pNBS-HGG). Alterations in the gene itself include amplification, mutation, or both[24, 50-52, 55, 56, 60-63]. Experimentally, overexpression of wild-type (WT) or mutant PDGFR $\alpha$ confers a growth advantage to astrocytes, an effect that is diminished by the introduction of the adenosine triphosphate-competitive inhibitors crenolanib or dasatinib. PDGFR $\alpha$ mutants drive glioma formation in vivo[24, 25], with murine-derived HGGs recapitulating critical features of the human disease such as histopathologic characteristics and expression profiles[24]. In an effort to target PDGFR therapeutically, pediatric trials using dasatinib, crenolanib, or imatinib have been launched[64-66]. Unfortunately, the benefit derived from selective RTK inhibitors may be marginal at best. pHGGs show evidence of intratumoral heterogeneity, with some cells co-amplifying multiple RTK genes or discrete cell populations within the same tumor amplifying different genes, suggesting that resistant populations are likely to be present even before treatment with targeted agents[51,61].

Both PDGFRA and EGFR are part of the RTK-Ras-PI3K signaling cascade, which is altered in nearly $90 \%$ of aHGGs. Additionally, $80 \%-90 \%$ of adult tumors show evidence of RB and p53 pathway dysregulation[42-44, 67]. For this reason, many of the first genetic $\mathrm{pHGG}$ studies focused on these same networks.

In adults, the most commonly targeted components of the RTK-Ras-PI3K axis downstream of RTKs include activation of PI3K itself, or loss of function of phosphatase and tensin homolog (PTEN), the main negative regulator of PI3K signaling, or NF1, a negative regulator of Ras-mediated signaling[42-44]. Activation of PI3K signaling caused by mutations of PIK3CA, encoding the catalytic p $110 \alpha$ subunit of PI3K, or $P I K 3 R 1$, encoding the regulatory subunit of PI3K, is usually present in mutually exclusive patterns, occurring in approximately $20 \%$ of aHGGs and in a similar frequency of pHGG, including DIPG[8-11, 42, 62, 68-72]. The PTEN tumor suppressor is located on chromosome 10q. It remains unclear whether all tumors with loss of chromosome 10q are targeting PTEN loss of function when a WT PTEN allele is still retained. However, there are examples in experimental systems where PTEN haploinsufficiency contributes to tumorigenesis. Loss of heterozygosity $(\mathrm{LOH})$ of chromosome $10 \mathrm{q}$, with or without concurrent PTEN mutation, is very frequent in adult glioblastoma, with $10 \mathrm{q}$ LOH in approximately $80 \%$ and PTEN mutation in $25 \%-40 \%$, whereas the frequency is significantly lower in pHGGs, with $10 \mathrm{q}$ LOH in approximately $30 \%$ and PTEN mutation in less than $5 \%-15 \%[8,42,50-53,55,56,60-63,69,73-75]$.

RB pathway dysregulation is common in both pNBS-HGGs and DIPG. The $C D K N 2 A$ locus codes for 2 tumor suppressors, p16INK4a and ARF[45]. Notably, homozygous deletion of $C D K N 2 A / B$ appears to be almost exclusive to pNBS tumors and largely absent in DIPGs[50-52, 55, 56, 60-63, 76]. In contrast, amplification of $C D K 4 / 6$ or $C C N D 1 / 2 / 3$ is found in approximately $30 \%$ of DIPG $[51,55,61]$. Therapeutic inhibition of this cyclin-CDK complex, using PD-0332991, a highly-selective non- 
adenosine triphosphate-competitive CDK4/6 inhibitor, significantly increased survival in a murine model of DIPG, both as a single agent or following irradiation[77].

TP53 mutations occur in up to $35 \%$ of pNBS-HGGs (range: $18 \%-35 \%$ ) and appear to be more common in DIPGs (40\%- 50\% of cases) $[8-11,54,62,67,69,73-75$, 78, 79].

From the aforementioned data, we can conclude that although the 3 main signaling pathways affected in aHGG are also affected in $\mathrm{pHGG}$, pediatric and adult tumors differ regarding the most frequently mutated effectors.

Despite some common copy number imbalances such as $13 \mathrm{q}$ and $14 \mathrm{q}$ loss in approximately one-third of patients with HGG regardless of age or location, aHGG and pHGG also exhibit a unique constellation of gains and losses that distinguish one from the other, and the same can be said for DIPGs and pNBS-HGGs[50-52, 55, 56, 60-63]. This suggests that unique combinations of genetic drivers underlie adult and pediatric tumorigenesis, and among childhood HGG, DIPG and pNBS-HGG tumorigenesis.

Transcriptional analysis of tumors supports similar conclusions. Clustering of gene expression signatures from aHGGs identifies 3-4 major gene expression subgroups, with the most robust distinction between proneural and mesenchymal subgroups[32, 8082]. Unlike the WNT and sonic hedgehog subgroups of medulloblastoma (another type of pediatric brain tumor)[83], the mutations associated with particular HGG subgroups are much less consistent. These same subgroups were identified in pHGGs by unsupervised comparisons, showing a clear relationship in the gene expression signatures of gliomas among different age groups and locations. However, supervised comparisons revealed expression signatures that distinguished adult from pediatric tumors, and within childhood glioma, DIPGs from pNBS-HGGs[51, 52, 55, 61, 84, 85].

Because biopsy on patients with DIPG is not routinely performed in the United States, most research material is acquired at autopsy from irradiated patients. The vast majority of these samples are designated grade IV[86]. In contrast, clinicians in France regularly perform pretreatment biopsies, and although most samples were HGGs, some were classified as low grade[87], raising the possibility that there may be a low-grade to high-grade malignant transformation in the genesis of DIPG. Importantly, there is a high degree of similarity in the copy number imbalances and expression signatures from DIPGs collected as biopsy samples before treatment and those collected at autopsy, which were treated by radiation in most cases, with or without chemotherapy [51,61]. Furthermore, expression signatures of pediatric brainstem LGGs are much more closely related to non-brainstem LGG and not to DIPGs, emphasizing a different etiology underlying genesis of LGG and HGG arising in the brainstem[51].

This body of work established the concept that oncogenic events driving pediatric HGG were different from those arising in adults. This appreciation, however, was not fully cemented until early 2012, with the discovery of recurrent histone mutations in pHGG (Figure 1-2). As the first reports of histone mutations in human cancer, these 
A.

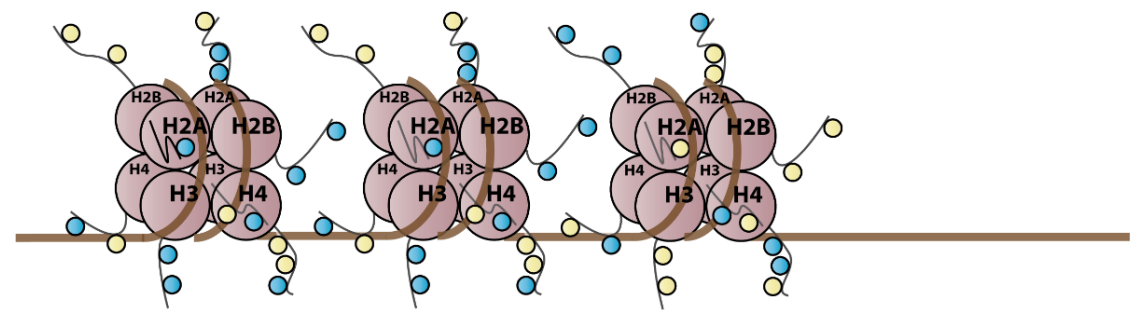

B.

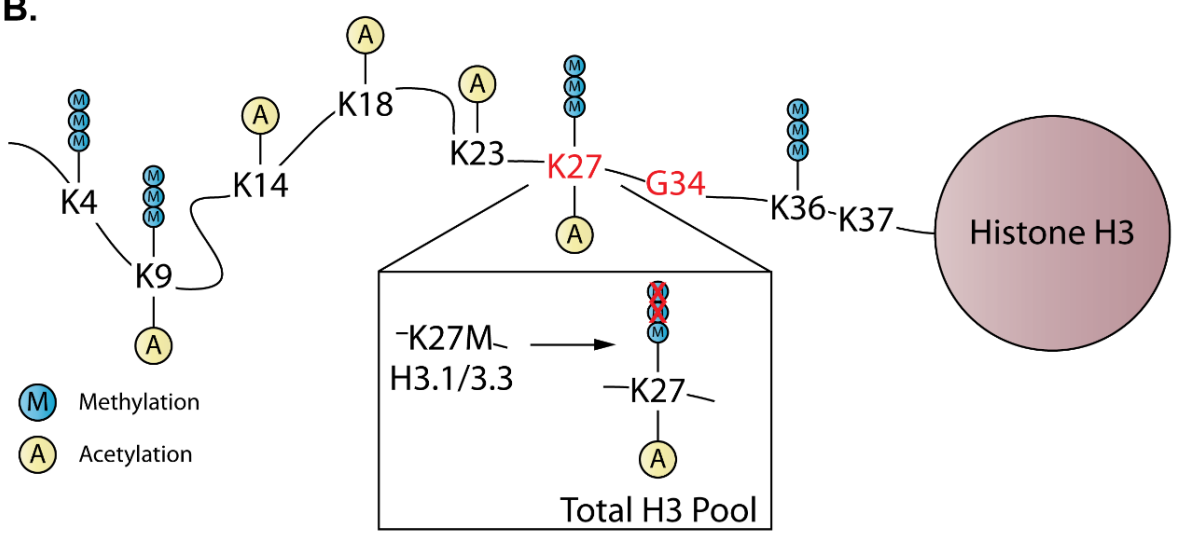

C.

\begin{tabular}{llllll} 
Gene & Histone & Mutation & Effect & DNA Methylation & Gene Expression \\
\hline H3F3A & H3.3 & p.K27M & Decreased total H3K27me2/3 & Changed & Changed \\
HIST1H3B & H3.1 & p.K27M & Decreased total H3K27me2/3 & Changed & Changed \\
H3F3A & H3.3 & p.G34R/V & No change in total H3K27me2/3 & Changed & Changed
\end{tabular}

Figure 1-2. Hot-spot histone mutations occur in nearly $80 \%$ of DIPGs and $\sim 35 \%$ of NBS-HGGs.

(A) The basic unit of chromatin is the nucleosome, in which the DNA is wrapped around a histone octamer consisting of 2 copies of $\mathrm{H} 2 \mathrm{~A}, \mathrm{H} 2 \mathrm{~B}, \mathrm{H} 3$, and $\mathrm{H} 4$. The N-terminal tails of histones undergo post-translational modifications (PTMs), represented by yellow and blue circles, which in turn alter chromatin accessibility and recruitment of effector proteins, together influencing transcriptional permissiveness. This is accomplished because PTMs can (1) themselves alter the strength of DNA-histone interactions and (2) facilitate recruitment of chromatin remodeling complexes or histone PTM-binding effector proteins. (B) p.K27M substitutions occur in histone H3.1 and H3.3; p.G34R/V substitutions occur in H3.3. Histone H3.1/3.3 p.K27M exerts a dominant effect, preventing the accumulation of $\mathrm{H} 3 \mathrm{~K} 27 \mathrm{me} 2 / 3$ on the wild-type histone $\mathrm{H} 3$ expressed in the same cell. p.G34R/V mutations do not exert the same effect on H3K27me3, but p.K27M and p.G34R/V histone mutations are associated with distinct genome-wide DNA methylation and gene expression tumor signatures. (C) Table summarizing the functional consequences of histone mutations. 
publications implicated novel mechanisms in pHGG tumor biology that are not found to play a significant role in the adult disease.

Whole-genome sequencing of 7 DIPGs and matched germline DNA, as part of the St. Jude Children's Research Hospital-Washington University Pediatric Cancer Genome Project, identified somatic mutations in $H 3 F 3 A$ leading to a p.K27M substitution in histone $\mathrm{H} 3.3$ in 4 of 7 cases. A fifth case contained an analogous mutation in HIST1H3B yielding a p. K27M alteration in histone H3.1. Subsequent targeted sequencing of all 15 genes encoding histone $\mathrm{H} 3$ in a larger cohort of $\mathrm{pHGG}$ found $\mathrm{p} . \mathrm{K} 27 \mathrm{M}$ somatic mutations in $78 \%$ of DIPGs: $60 \%$ were mutations in $H 3 F 3 A$ and $18 \%$ in $H I S T 1 H 3 B$ [88]. Similar frequency of histone $\mathrm{H} 3$ mutation was found in an independent cohort; however, the proportion of $H 3 F 3 A$ and $H I S T 1 H 3 B$ mutations varied between the groups, likely owing to patient age, with $H I S T 1 H 3 B$ mutations arising in younger children[88, 89]. In pNBSHGG, H3F3A and HIST1H3B p.K27M substitutions were found in $19 \%$ and $3 \%$ of cases, respectively. Additionally, $14 \%$ of pNBS-HGGs harbored somatic mutations in $\mathrm{H} 3 \mathrm{~F} 3 \mathrm{~A}$ leading to p.G34R substitution, whereas no such alteration was identified in any DIPG. Most of the DIPG samples evaluated were collected at autopsy. However, of 8 DIPG samples collected from patients who had not received adjuvant therapy, 7 contained p.K27M substitutions. Hence, histone $\mathrm{H} 3$ alterations were not necessarily secondary to therapy[88].

Collaborating groups in Canada and Germany performed whole-exome sequencing of 48 pNBS-HGGs and identified p.K27M, p.G34R, and p.G34V alterations in $19 \%, 10 \%$, and $2 \%$ of cases, respectively, with all changes affecting $H 3 F 3 A$ encoding the histone $\mathrm{H} 3.3$ variant. Additional targeted sequencing of the $H 3 F 3 A$ locus in more than 700 samples of gliomas of various grades from patients of different ages revealed these mutations to be exclusive to high-grade tumors and significantly enriched in pediatric cases. Of the 11 adult cases harboring $H 3 F 3 A$ mutation, all were missense substitutions of G34, and the majority were identified in young adults aged 20-30 years. The specific histone mutation was associated with anatomical location. p.K27M mutations occurred in tumors involving midline structures (such as the brainstem, cerebellum, and thalamus), whereas p.G34R/V mutations occurred in non-midline supratentorial lesions (Figure 1-3) [8,69].

Nucleosomes are the basic unit of chromatin, in which DNA is wrapped around a nucleosome core composed of a histone octamer with 2 copies each of histones H2A, $\mathrm{H} 2 \mathrm{~B}, \mathrm{H} 3$, and $\mathrm{H} 4 . \mathrm{H} 3 \mathrm{~F} 3 \mathrm{~A}$ and $\mathrm{HIST} 1 \mathrm{H} 3 \mathrm{~B}$ code for the 2 histone $\mathrm{H} 3$ variant isoforms H3.3 and H3.1, respectively. There are 3 isoforms of histone H3. Histone H3.1 and H3.2 are encoded by 10 and 3 separate genes, respectively, and are synthesized during $\mathrm{S}$ phase of the cell cycle to package newly replicated DNA. Histone H3.3 is synthesized throughout the cell cycle and selectively incorporated into promoter regions of active genes, and through interactions with ATRX (alpha thalassemia/mental retardation syndrome X-linked) and DAXX (death domain-associated protein 6), into pericentromeric heterochromatin and sub-telomeric regions[90]. Notably, recurrent ATRX loss-of-function mutations were found in approximately one-quarter of $\mathrm{pHGG}$, which were also associated with alternative lengthening of telomeres. All tumors with $H 3 F 3 \mathrm{~A}$ 
A.

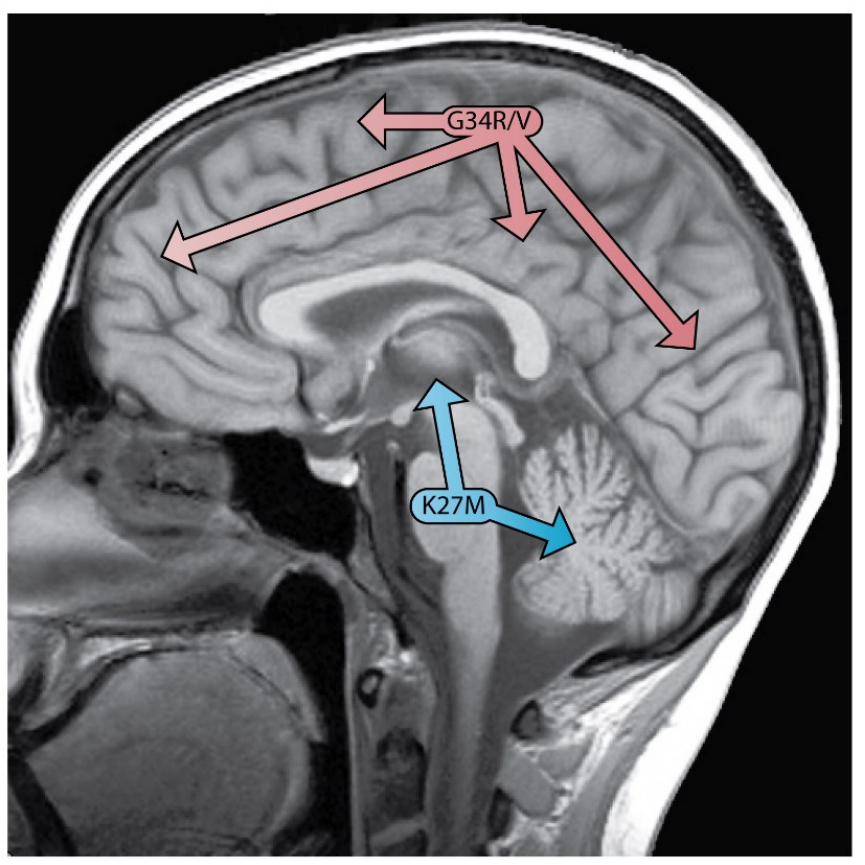

B.

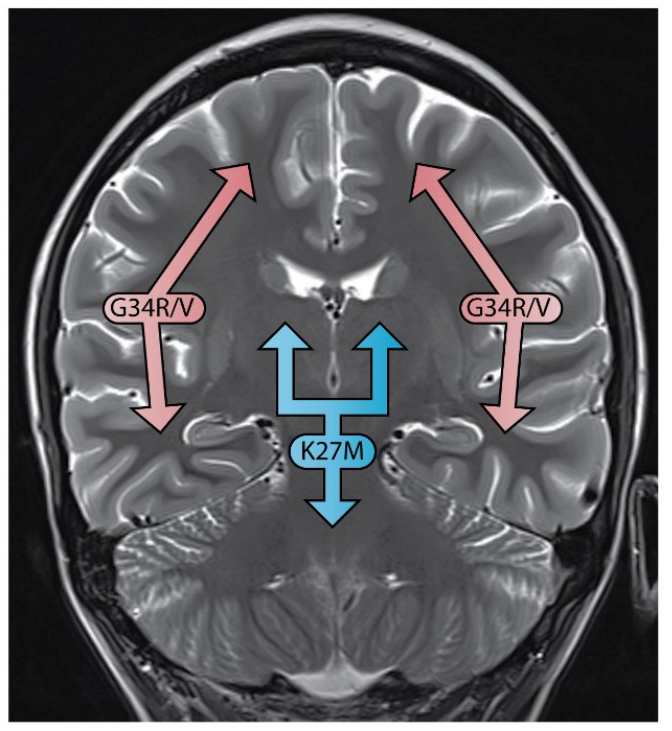

Figure 1-3. Histone mutations are strongly associated with anatomical location. Midsagittal (A) and coronal (B) magnetic resonance images demonstrate that p.K27M mutations occur predominantly in tumors involving midline structures (the brainstem, cerebellum, and thalamus), whereas p.G34R/V mutations mainly occur in tumors arising in the cerebral hemispheres. 
p.G34R/V mutation carried concomitant $A T R X$ mutations, suggesting synergy between the 2 mutations[69].

All identified histone mutations occur in the N-terminal tails of histones, unstructured regions that undergo extensive post-translational modification. These modifications in turn facilitate recruitment of effector proteins that regulate transcriptionally active or silent chromatin states[91].

All histone $\mathrm{H} 3$ mutations in $\mathrm{pHGG}$ were heterozygous, and in any individual tumor, only 1 of 15 genes encoding histone $\mathrm{H} 3$ was mutated. This pattern clearly indicates a dominant gain-of-function effect.

Advances in microarrays and next-generation sequencing technologies have provided unprecedented insight into pHGG biology. These studies, both large and small scale, genome-wide and targeted, have given today's researchers and clinicians a better understanding of pHGG than ever before."

\section{Closing Introductory Remarks}

One hurdle shared by all researchers in studying pHGG is the relative lack of available biomaterial, with only several hundred new cases diagnosed in the United States each year[12]. This dissertation begins with the analysis of a cohort of 127 tumors by next-generation sequencing. The next chapter is the publication that resulted from those efforts with the subsequent chapter being an extension of one of the main findings from that report. 


\section{CHAPTER 2. THE GENOMIC LANDSCAPE OF DIFFUSE INTRINSIC PONTINE GLIOMA AND PEDIATRIC NON-BRAINSTEM HIGH-GRADE GLIOMA*}

The second chapter of this dissertation will be the publication that resulted from our analysis of $127 \mathrm{pHGGs,} \mathrm{both} \mathrm{DIPGs} \mathrm{and} \mathrm{NBS-HGGs[8].} \mathrm{Quoted} \mathrm{text} \mathrm{is} \mathrm{taken} \mathrm{from}$ the manuscript. This study was a collaborative effort between our laboratory and Dr. Jinghui Zhang's group in the Department of Computational Biology. I shared first authorship with Dr. Gang Wu who spearheaded the bioinformatic analysis of sequence data. My contribution was the functional study of the two primary genetic alterations described below.

"Although childhood and adult HGGs share related histopathological characteristics, adult HGGs arise predominantly in the cerebral cortex, whereas childhood HGGs more frequently involve a broader spectrum of locations. There are also substantial differences in the molecular features of pediatric and adult HGGs[43, 44, 50$52,55,56,61,63,69,84,92]$. Histone $\mathrm{H} 3$ gene $(H 3 F 3 A$ and HIST1H3B) mutations encoding p.Lys27Met are frequent in DIPGs, which arise in the brainstem almost exclusively in children, and in pediatric HGGs in midline structures such as the thalamus and cerebellum, whereas histone $\mathrm{H} 3$ gene mutations encoding p.Gly34Arg or p.Gly34Val occur in pediatric HGGs of the cerebral cortex [69, 88, 89, 93]. In contrast, histone H3 gene mutations are extremely rare in adult HGGs[69]. HGGs arising in infants younger than 3 years of age have a better prognosis and a lower frequency of TP53 mutations, suggesting that there may be age-dependent subgroups of HGG, even within the pediatric population[94]. Thus, the selective pressures driving gliomagenesis in children vary with age and anatomical site.

To more comprehensively understand the pathways driving childhood glioma, we analyzed the genomic landscape of HGGs from 118 pediatric cases (127 tumors, 108 matched to germline DNA) consisting of 57 DIPGs and 70 NBS-HGGs by wholegenome $(n=42)$, whole-exome $(n=80)$ and transcriptome $(n=75)$ sequencing. A total of 39,590 sequence mutations, including single-nucleotide variations (SNVs) and small insertions or deletions (indels), and 2,039 structural variations were found by wholegenome sequencing, and an additional 2,600 sequence mutations and 138 structural variations were found by exome sequencing and transcriptome sequencing, respectively. Overall, the cohort showed a median background mutation rate of $9 \times 10^{-7}$ mutations per base and a median of 22 structural variations per genome. All SNVs and structural variations found by whole-genome sequencing were verified experimentally by independent sequencing methods.

\footnotetext{
${ }^{*}$ Reprinted with permission. Wu, G., Diaz, A.K., et al., The genomic landscape of diffuse intrinsic pontine glioma and pediatric non-brainstem high-grade glioma. Nat Genet, 2014. 46(5): p. 444-50.
} 
Among recurrent mutations in pediatric $\mathrm{HGG}$, the most frequently mutated gene not previously identified in cancer was $A C V R 1$ (also known as $A L K 2$ ) encoding a bone morphogenetic protein (BMP) type I receptor (Figures 2-1 and 2-2). Clonal missense ACVR1 mutations were found exclusively in DIPGs (32\%) and were significantly associated with younger age, longer survival time and the presence of HIST1H3B mutation encoding p.Lys27Met $(P<0.0000001)$ or with $P I K 3 C A$ or $P I K 3 R 1$ mutations $(P<0.005)$ (Figures 2-1 and 2-2). Four of these somatic $A C V R 1$ mutations were the same as germline mutations previously identified in the autosomal dominant syndrome fibrodysplasia ossificans progressiva (FOP), in which aberrant cellular differentiation drives progressive heterotopic ossifications [95, 96]. All residues affected by mutation in DIPGs clustered around either the inhibitory glycineserine-rich (GS) domain or the ATP-binding pocket of the kinase domain and would be expected to shift the kinase to an active conformation (Figure 2-2)[97]. Indeed, mutations affecting these residues induced a weak gain of function[97, 98]. A previous study showed that the ACVR1 mutation encoding p.Arg206His caused a ventralized phenotype in zebrafish embryos, an indicator of BMP pathway activation[99]. We tested all of the $A C V R 1$ mutations found in DIPG using this assay. Zebrafish embryos injected with wild-type human $A C V R 1$ mRNA displayed a mild dorsalized phenotype consistent with BMP pathway inhibition, whereas injection with mRNA for all six $A C V R 1$ mutants resulted in varying degrees of ventralization, with partial to complete loss of head and dorsal structures (Figure 2-2b, c). A moderate dose of LDN-193189, a highly selective antagonist of the BMP pathway[99, 100], partially reversed the ventralization effects induced by $A C V R 1$ mutants, as demonstrated by the rescue of dorsal head structures for the Arg258Gly, Gly328Glu, Gly328Trp and Arg206His mutants and the reduced severity of ventralization for the Gly356Asp and Gly328Val mutants (Figure 2-2c). Expression of ACVR1 mutants in mouse primary astrocyte cultures caused increased levels of phosphorylated SMAD1 and SMAD5 (SMAD1/5), a downstream indication of active BMP signaling, with varying magnitude (Figure 2-2d). LDN-193189 also effectively blocked signaling to phosphorylated SMAD1/5 downstream of mutant ACVR1 in primary astrocytes.

Recurrent and clonal activating mutation of $A C V R 1$ in $32 \%$ of DIPGs provides strong evidence that $A C V R 1$ is an oncogenic driver in this disease. However, germline $A C V R 1$ mutation in the genetic syndrome FOP is not associated with cancer predisposition, indicating that $A C V R 1$ mutation likely provides a selective advantage in the presence of other critical mutations rather than driving tumor initiation. Consistent with this hypothesis, all six of the DIPG-associated ACVR1 mutants failed to render Tp53-null mouse astrocytes tumorigenic when implanted into the brain (data not shown). BMP signaling is associated with contrasting effects dependent on context, driving astrocytic differentiation or the proliferation of early hindbrain progenitors[101]. In tumors, BMP signaling induced the differentiation of medulloblastoma[102] but drove either a differentiation or proliferation response in glioblastoma stem cells, related in part to the epigenetic state of the cell[103,104]. These context-dependent consequences likely drive the exclusive association between $A C V R 1$ mutation and DIPG. 


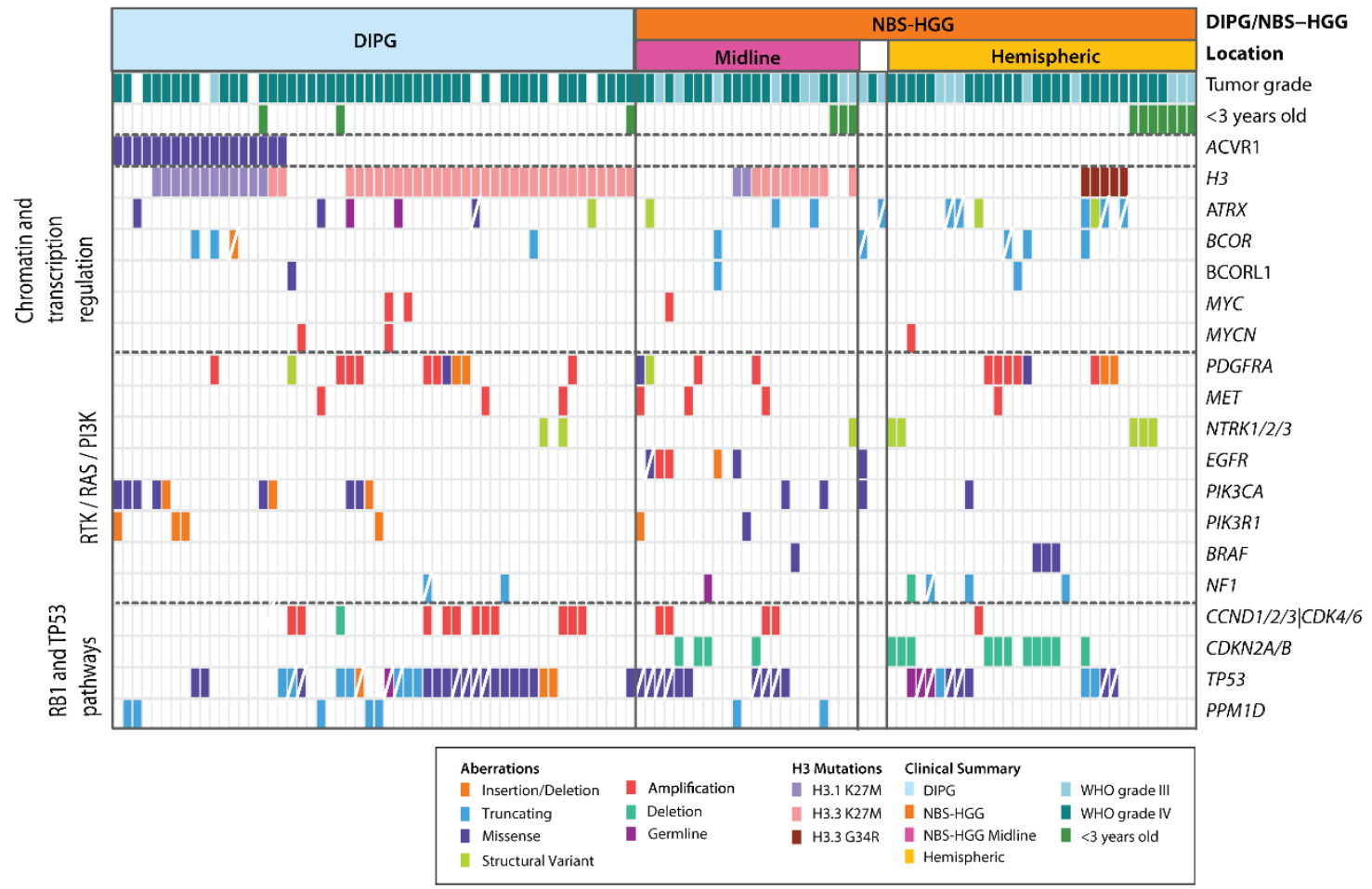

Figure 2-1. Recurrent genetic alterations in pediatric HGG.

Genetic alterations detected in 19 genes, including $A C V R 1$ and the genes most recurrently mutated in the pathways indicated on the left, are displayed. A diagonal white line indicates loss of the wild-type allele or a male case for $A T R X$, which is X linked. $H 3 F 3 A$ (H3.3) and HIST1H3B (H3.1) mutations are grouped together in the category histone H3. Structural variants involving NTRK1, NTRK2 or NTRK3 and copy number variants (CNVs) of components of the CCND1, CCND2, CCND3, CDK4 or CDK6 G1 checkpoint complex are grouped together. Tumor subgroup (DIPG or NBS-HGG), location of NBSHGG (midline versus cerebral hemisphere) and World Health Organization (WHO) tumor grade are indicated. White boxes for location or tumor grade indicate information not available. Mutations for 112 HGGs are shown. Four hypermutator samples and 11 samples for which only RNA-seq data were available were excluded from this summary. 
Figure 2-2. $\quad A C V R 1$ mutations in DIPG activate BMP signaling. (A) Missense $A C V R l$ substitutions in DIPG were clustered in the GS or kinase domains. Each red circle indicates a DIPG carrying the specified alteration, and an asterisk indicates alterations previously found as germline mutations in individuals with FOP. The extracellular domain (EC) and transmembrane domain (TM) were not affected by mutations. (B) Human ACVR 1 mutants ventralize zebrafish embryos. The graph shows the percentage of embryos exhibiting a dorsalized or ventralized phenotype. Embryos injected with wild-type $A C V R 1$ mRNA (WT) showed a dorsalized phenotype, whereas embryos injected with mutant $A C V R 1$ mRNA showed a ventralized phenotype (increasing severity from left to right). The Arg258Gly mutant had the least severe effect, resulting only in the V3-V4 ventralized phenotype, whereas the Gly328Val mutant had the most severe effect, with $90 \%$ of embryos showing the V5 ventralized phenotype. The number of embryos examined is shown on top. (C) Representative phenotype images of zebrafish embryos injected with the indicated $A C V R 1$ mRNA. Untreated embryos expressing the Arg258Gly, Gly328Glu, Gly328Trp and Arg206His mutants have few to no dorsal structures, and embryos expressing the Gly356Asp and Gly328Val mutants are more severely affected. Treatment with LDN- 193189 reversed the ventralization effects of mutant ACVR1, as demonstrated by the partial rescue of dorsal structures (e.g., the head) in embryos expressing the Arg258Gly (100\%, $\mathrm{n}=20)$, Gly328Asp (83\%, $\mathrm{n}=23)$, Gly328Trp $(100 \%, \mathrm{n}=20)$ and Arg206His $(100 \%, \mathrm{n}=27)$ mutants and the reduced severity of ventralization showing a decrease in the proportion of embryos with the V5 phenotype without the formation of dorsal structures for embryos expressing the Gly356Asp (89\%, $\mathrm{n}=27)$ and Gly328Val $(49 \%, \mathrm{n}=24)$ mutants. Scale bar, $200 \mu \mathrm{m}$. (D) ACVR1 mutations drive increased levels of phosphorylated SMAD1/5 (pSMAD1/5) in primary astrocyte cultures. Protein blots are shown for lysates of primary astrocytes isolated from the brainstem of neonatal mice with conditional knockout of Tp53 transduced with retroviruses expressing Flag-tagged wild-type ACVR1 or the indicated mutants and serum starved for $2 \mathrm{~h}$. Quantification of the ratio of phosphorylated SMAD to total SMAD normalized to the empty vector control is shown below. 
A.

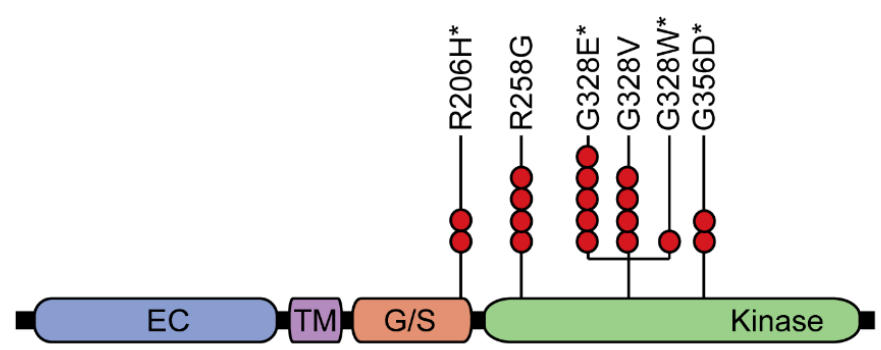

B.

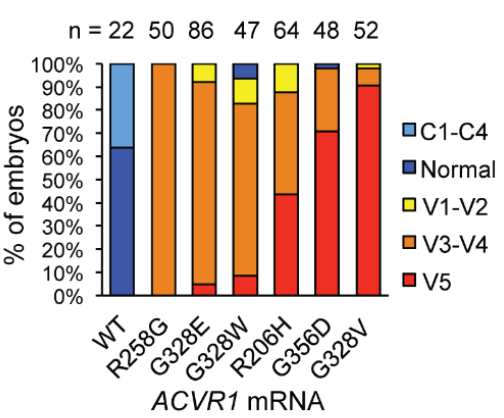

D.

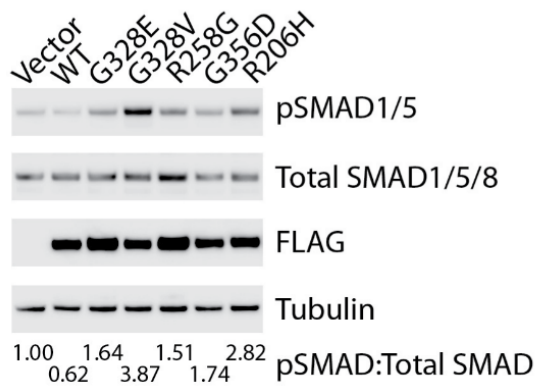

c.

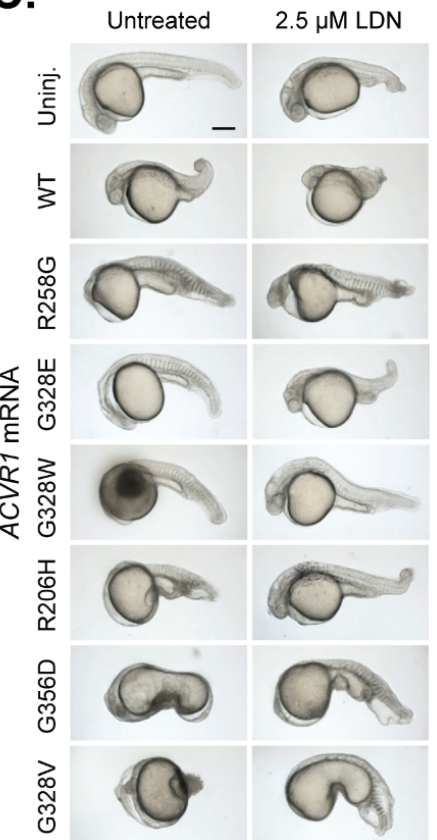


Principal-component analysis using the 1,000 most variable probe sets showed that HGG samples clustered by tumor location with no segregation of DIPGs by $A C V R 1$ mutation status. Genes involved in the regulation of immune system processes showed significantly different expression in DIPGs with and without ACVR1 mutation (Fisher's exact test, $P=0.0002$, false discovery rate $(\mathrm{FDR})=0.26 \%$ ).

Structural variants generating fusion genes were common and were identified in $47 \%$ of pediatric HGGs, in equal proportions of DIPGs and NBS-HGGs, from transcriptome and whole-genome sequencing data. Gene fusions involving the kinase domain of each of the three neurotrophin receptor (NTRK) genes and five different Nterminal fusion partners were identified in $4 \%$ of DIPGs and $10 \%$ of NBS-HGGs. Notably, 40\% (4/10) of NBS-HGGs in children younger than 3 years old contained an NTRK fusion gene (Figures 2-1 and 2-3). The NTRK receptors transduce a wide range of developmental signals in the nervous system, including the induction of neurite outgrowth, differentiation and neuronal survival or death $[105,106]$. NTRK fusion genes have recently been identified at low frequency in low-grade pediatric astrocytomas as well as in adult glioblastomas[107-109]. Two of the five NTRK fusions found in our cohort, TPM3-NTRK1 and ETV6-NTRK3, were previously identified in other cancer types and shown to be oncogenic[110-114].

To test the ability of NTRK fusion genes to drive glioma formation, we implanted Tp53-null primary mouse astrocytes transduced with retrovirus expressing Flag-tagged human TPM3-NTRK1 or BTBD1-NTRK3 into mouse brain. Both NTRK fusions induced high-grade astrocytomas with very short latency and complete penetrance (Figure 2-3b). The resulting tumors showed elevated levels of phosphorylated AKT and p42/44 mitogen-activated protein kinase (MAPK), downstream indicators of phosphoinositide 3-kinase (PI3K) and MAPK pathway activation.

Although NTRK-activating fusions were specifically found at high frequency in tumors from infants, activation of receptor tyrosine kinase (RTK)-RAS-PI3K signaling through other mutations was frequent across the entire cohort, occurring in $69 \%$ of DIPGs and 67\% of NBS-HGGs (Figure 2-1). In contrast to previous reports detecting EGFRvIII expression in pediatric HGG[58, 59], we only detected EGFRvIII expression in 1 of the 85 tumors analyzed by whole-genome sequencing or RNA sequencing (RNAseq).

In addition to recurrent histone $\mathrm{H} 3$ gene, ATR $X$ and SETD2 mutations reported previously[ $69,88,89,93,115]$, we also detected frequent mutations of other histone writers and erasers and of chromatin-remodeling genes (Figure 2-4). Interestingly, mutations affecting histone $\mathrm{H} 3$ writers were significantly more frequent in NBS-HGGs $(P=0.007)$, whereas frequencies of mutations affecting histone erasers were not significantly different in DIPGs and NBS-HGGs $(P=0.3)$. Although only ATRX mutations were highly recurrent, collectively, this group of genes involved in epigenetic regulation was targeted by mutation in $22 \%$ of DIPGs and $48 \%$ of NBSHGGs, excluding histone $\mathrm{H} 3$ gene mutations (Figure 2-4). These mutations were often concurrent with missense mutations in histone $\mathrm{H} 3$ genes. Indeed, including histone $\mathrm{H} 3$ 
A.

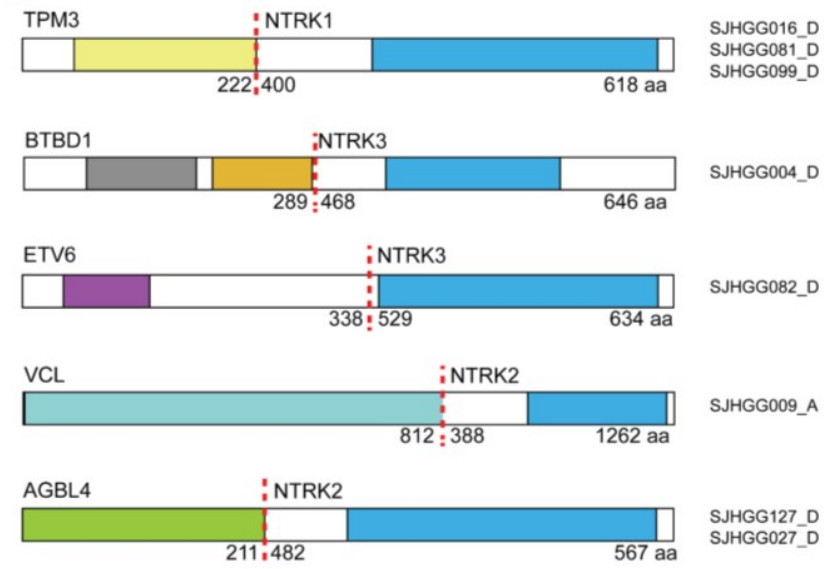

B.

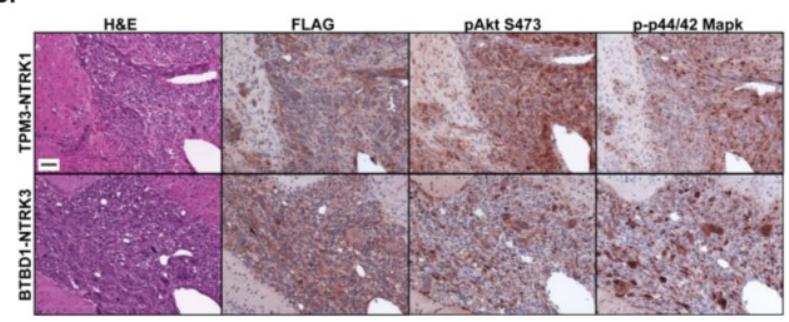

Figure 2-3. Three structural variants generate oncogenic chimeric NTRK fusion proteins.

(A) All fusions included the C-terminal kinase domain from NTRK1, NTRK2 or NTRK3 (blue). N-terminal fusion partners include the tropomyosin domain (yellow) of TPM3, an actin-binding protein fused to NTRK1, and the BTB/POZ dimerization domain (gray) and the Kelch domain (orange) from the topoisomerase I-interacting protein BTBD1 or the pointed protein-protein interaction domain (purple) of the ETS transcription factor ETV6 fused to NTRK3. The N terminus of the actin-binding protein vinculin (light blue; VCL) was fused to NTRK2, and the N terminus of the ATP/ GTP-binding protein AGBL4 (green) was fused to NTRK2. The functional carboxypeptidase domain of AGBL4 is not present in the fusion protein. For each fusion protein, the dashed red line shows the fusion point, with the amino acid positions of the $\mathrm{N}$-terminal and $\mathrm{C}$-terminal fusion partners at the breakpoint indicated. The number of amino acids in each full-length fusion protein is shown at the lower right of each fusion. (B) NTRK fusion proteins induce high-grade astrocytomas. Tp53-null mouse primary astrocytes isolated from neonatal cortex or brainstem were transduced with virus expressing Flag-tagged TPM3NTRK1 (top) or BTBD1-NTRK3 (bottom) and implanted into mouse brain.

Representative hematoxylin and eosin staining (of seven independent mice per construct) shows pleomorphic tumor cells, many with features of astrocytic differentiation and high mitotic activity. Tumors induced by BTBD1-NTRK3 showed the frequent presence of giant cells reminiscent of giant cell glioblastoma. Immunohistochemical analysis showed expression of Flag-tagged NTRK fusion proteins and elevated levels of phosphorylated AKT (pAKT) and 44/p42 MAPK (p-44/p42 MAPK) in tumor relative to surrounding normal tissue. Scale bar, $50 \mu \mathrm{m}$. 


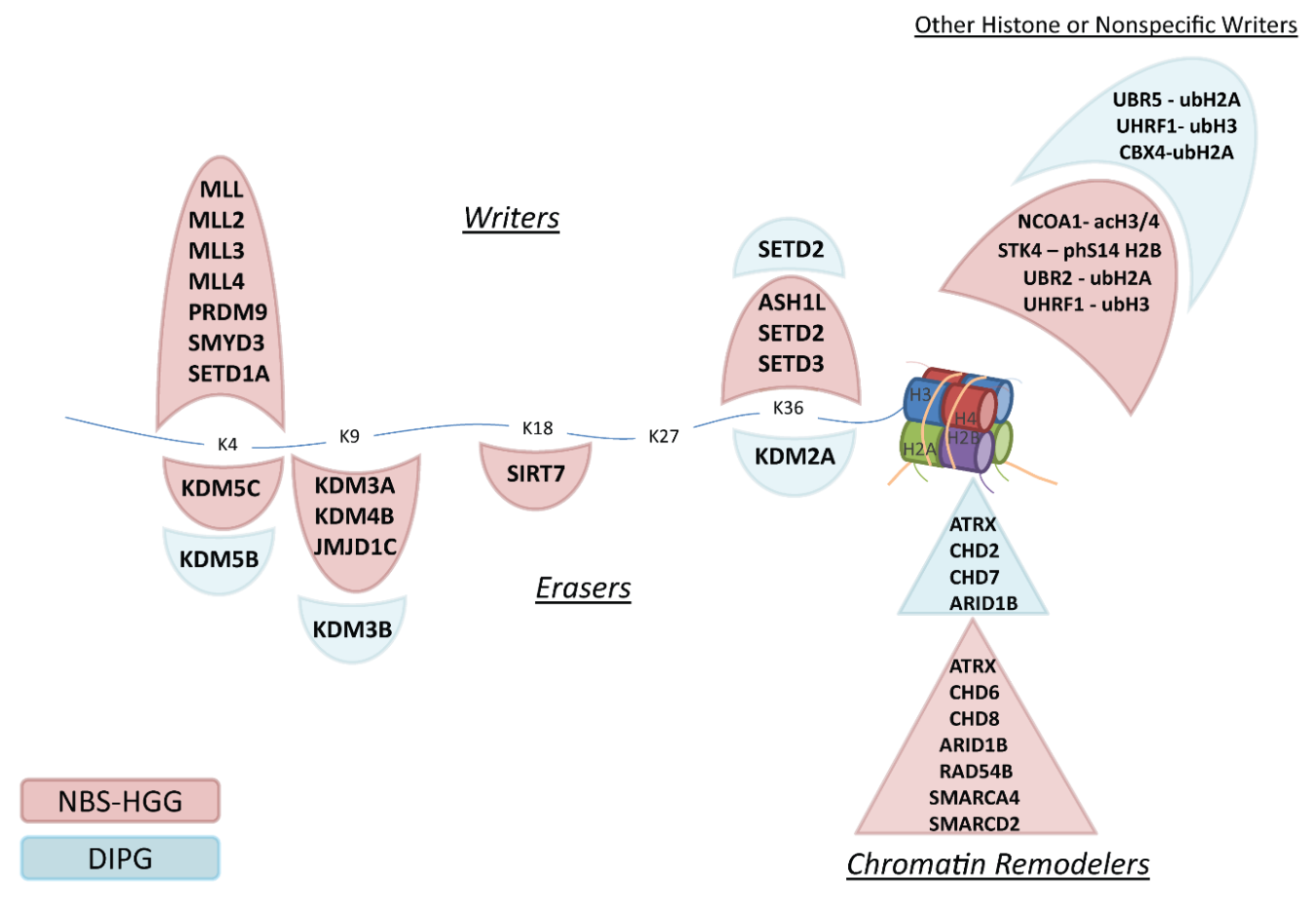

Figure 2-4. Pediatric HGG alterations in histone modifiers or chromatin regulators.

Alterations identified in NBS-HGG or DIPG are shown. Genetic alterations were identified in proteins that attach (writers; above) or remove (erasers; below) posttranslational modifications of lysine residues (Lys4, Lys9, Lys18, Lys27, Lys36) in the tail of histone $\mathrm{H} 3$, as well as proteins involved in modifications of other histones or chromatin remodelers. Notably, there were no alterations in writers or erasers of Lys27, which is directly mutated at high frequency in DIPG and, to a lesser degree, in NBSHGG. Ub, ubiquitination; ac, acetylation; ph, phosphorylation; H2, histone $\mathrm{H} 2$; H4, histone $\mathrm{H} 4$. 
gene mutations, $91 \%$ of DIPGs, $70 \%$ of midline NBS-HGGs and $48 \%$ of hemispheric NBS-HGGs contained mutations in histone genes and/or this subgroup of epigenetic regulators. p.Lys27Met histone $\mathrm{H} 3.3$ or $\mathrm{H} 3.1$ alteration results in a dominant loss of trimethylation at lysine 27 of histone $\mathrm{H} 3$ (H3K27me3) in the entire cellular pool of histone H3 proteins[116-119]. Mutations that modulate H3K27me3 levels by targeting components of the Polycomb-repressive complex 2 (PRC2) that methylates H3K27 or the H3K27 demethylases KDM6A and KDM6B are found in other tumor types[120-125]. However, there were no such mutations across the entire HGG cohort, including in DIPGs with wild-type histone H3 genes, supporting the unique selective advantage of p.Lys27Met alteration in pediatric HGG (Figure 2-4). Mutations in transcriptional regulators that affect the epigenetic landscape were also found, including focal amplifications of $M Y C$ and $M Y C N$, which encode transcription factors that act to amplify the levels of expressed genes across the genome[126, 127], and truncating mutations in the transcriptional corepressor genes BCOR and BCORL1 (Figure 2-1).

There was an enormous range in the complexity of the somatic mutations driving pediatric HGG. The ten NBS-HGGs in children under 3 years old showed significantly lower mutation rates than the rest of the cohort $(P<0.0001)$, suggesting that only a small number of driver mutations is required in tumors from this age group (Figures 2-5 and 2-6). ETV6-NTRK3 fusion was identified as one of only two non-silent alterations in SJHGG082, a glioblastoma from a 1-month-old subject. Notably, NTRK fusion genes, including two of the fusions found here, have been identified in multiple tumor types, including those from very young children such as congenital fibrosarcoma, as well as in papillary thyroid cancer, an adult disease[108, 110, 112-114]. The high frequency of NTRK fusion genes in NBS-HGGs from children younger than 3 years of age, the paucity of additional mutations in these tumors and the rapid tumor onset in our experimental glioma model strongly suggest that these fusion genes are potent oncogenic drivers in early postnatal brain tumor development.

Four tumors, 3 with matched normal DNA, were scored as hypermutators, with an extremely high background mutation rate, including more than 800,000 somatic mutations in SJHGG111 in which biallelic germline PMS2 mutations were identified (Figure 2-6). Subject SJHGG003 carried a heterozygous germline PMS2 mutation and developed a grade IV hemispheric malignant glioneuronal tumor (MGNT) and a separate DIPG. Although both tumors independently acquired different somatic inactivating mutations in the remaining $P M S 2$ allele, the basal mutation rate in the first tumor arising in this case, the MGNT (SJHGG003_D), was nearly 100-fold higher than in the DIPG that arose 2 years later (SJHGG003_A), demonstrating the potential range in the tumor mutation burden associated with inherited mutation in DNA mismatch repair genes (Figures 2-5 and 2-6). Hypermutator tumors were excluded from the evaluation of mutation frequency. Seven cases carried germline mutations in known cancer predisposition genes, including TP53, PMS2, MSH6 and NF1.

Thirteen tumors (31\%) analyzed by whole-genome sequencing had chromothripsis[128], identified by complex rearrangements with multiple interconnecting breakpoints corresponding to genomic segments with oscillating copy number states 
Figure 2-5. Circos plots showing the range of structural alterations in pediatric HGG.

Circos plots display the genome by chromosome in a circular plot and depict structural genetic variants, including DNA CNAs, intra- and interchromosomal translocations, and non-sequence mutations. Orange, loss of heterozygosity (LOH); red, amplification; blue, deletion. Sequence mutations in RefSeq genes: brown, missense SNV; red, indel; blue, splice-site SNV. Genes at structural variation breakpoints: pink, gene involved in inframe fusions; blue, others. Subject SJHGG003 carried a germline PMS2 mutation and developed two independent tumors, first a hemispheric malignant glioneuronal tumor (SJHGG003_D) and, 2 years later, a DIPG (SJHGG003_A). The SNVs and indels for these two tumors were too numerous to include. The hypermutator tumor with more than 800,000 somatic mutations had an extremely stable genome (SJHGG003_D), whereas the second tumor with approximately 100 -fold fewer SNVs carried typical genomic copy number and structural abnormalities seen in other HGGs (SJHGG003_A), thus demonstrating the broad range and complexity of mutations associated with germline PMS2 mutation. SJHGG016 D is an infant NBS-HGG with a TPM3-NTRK1 fusion and a very stable genome. SJHGG027_D is an NBS-HGG from a case with A-T, showing a relatively stable genome. This tumor sample was collected before radiotherapy. SJHGG004_D is a DIPG with chromothripsis driving BTBD1-NTRK3 fusion. SJHGG044_D is an NBS-HGG showing dramatic chromothripsis. For the examples of chromothripsis, the names of the genes disrupted by structural variants were too numerous to display. 

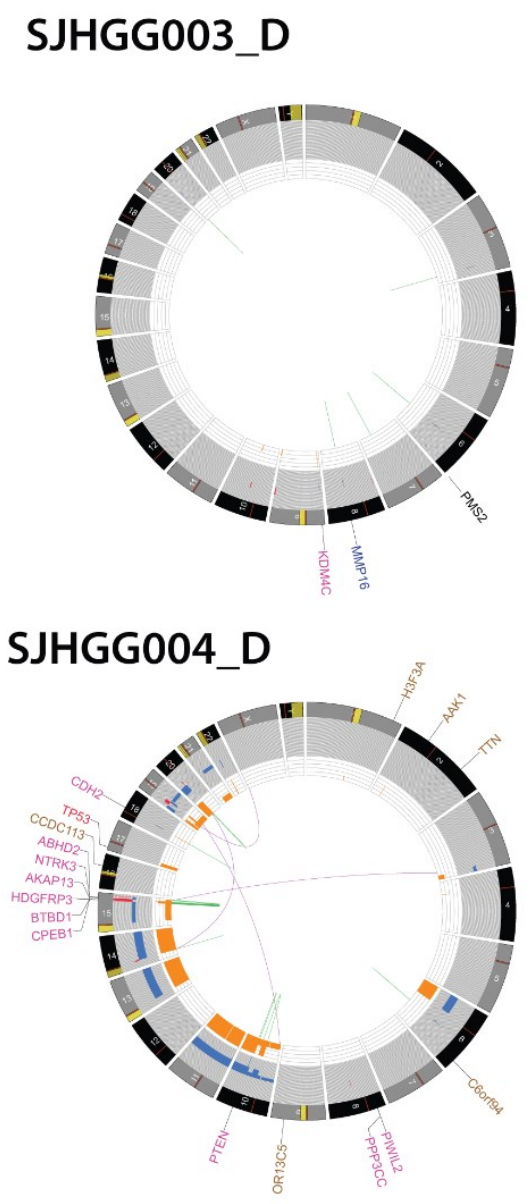

SJHGG027_D

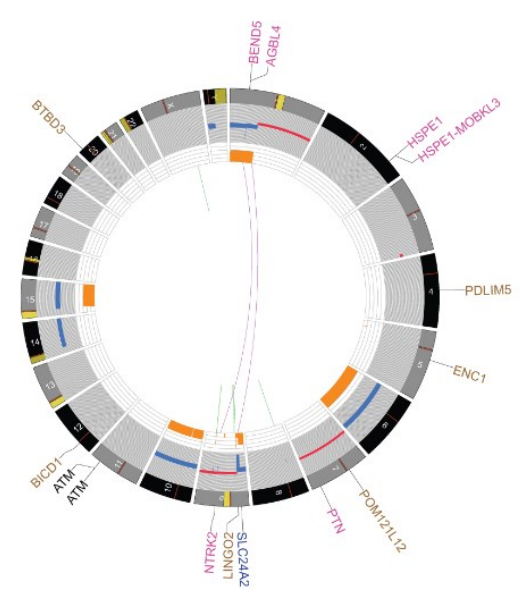

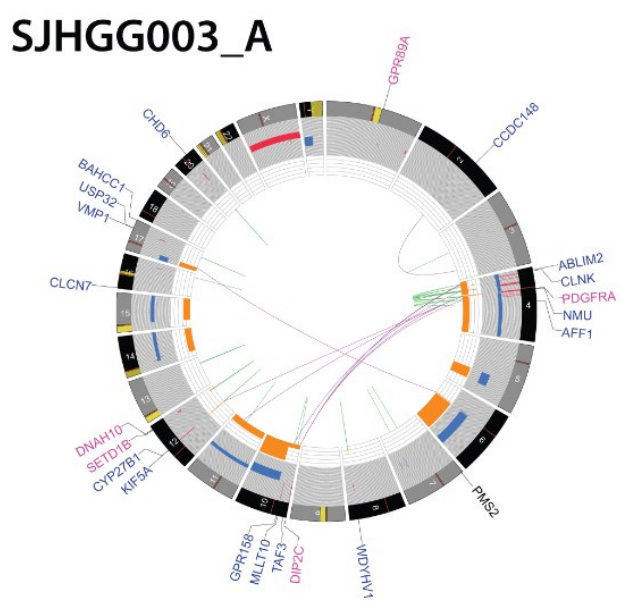

SJHGG016_D

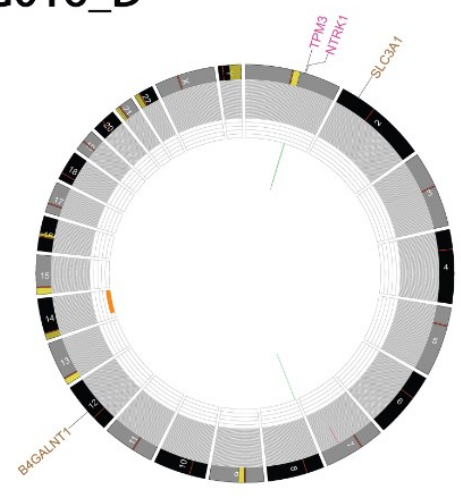

SJHGG044_D

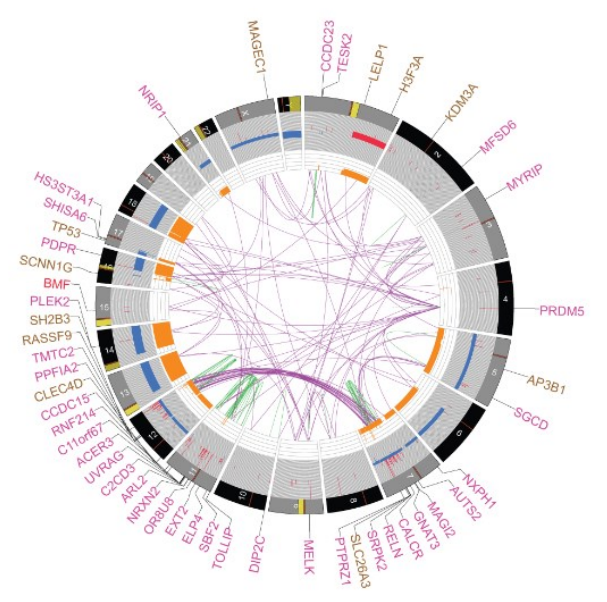




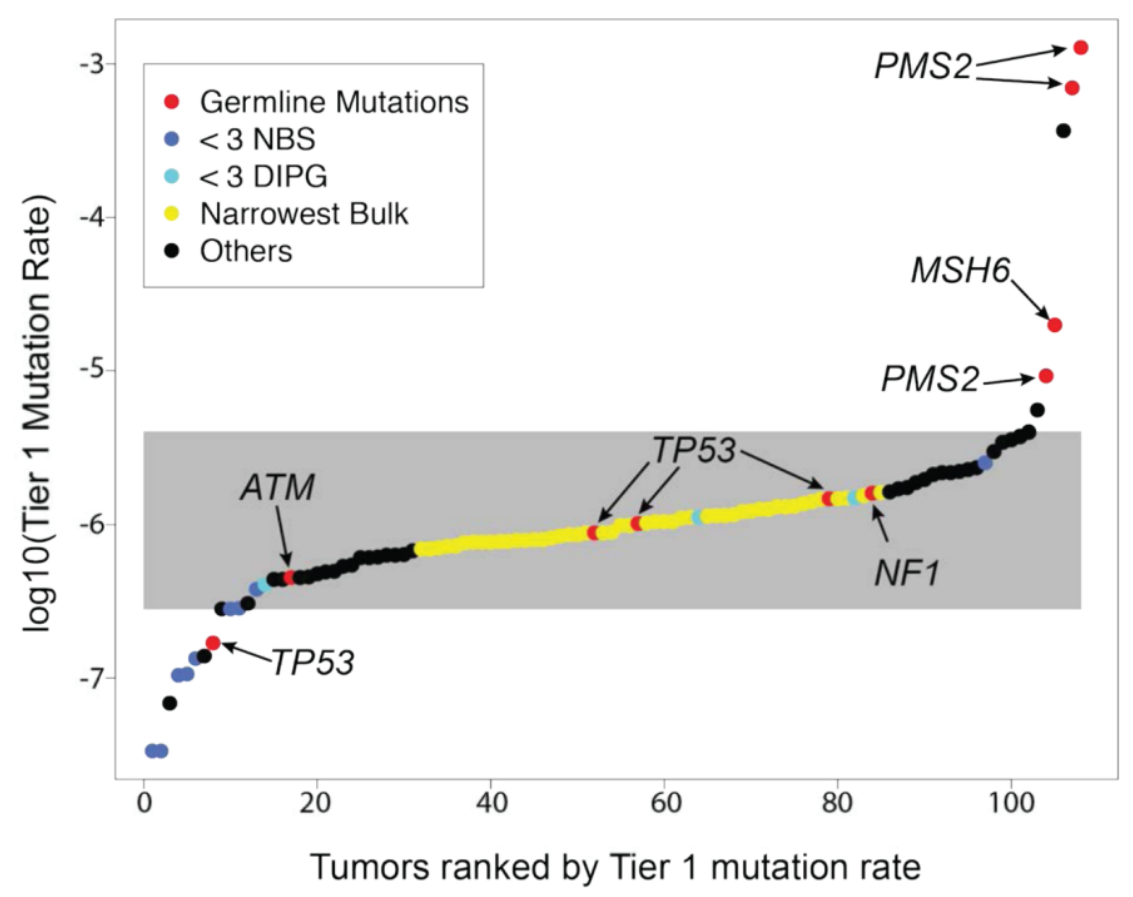

Figure 2-6. Outlier analysis of the tier1 mutation rate in all pediatric HGGs. Least median squares was used to find the narrowest interval containing at least $50 \%$ of the samples (shown in yellow) in terms of their tierl mutation rate. An approximate $95 \%$ prediction interval was determined by modeling this interval as the inter-quartile range of a normal distribution (shown by the gray box). Only samples with matched germline DNA were used for this analysis. The mutation rate is significantly lower in tumors from children less than 3 years of age than in the others $(p=0.0005823$, Wilcoxon rank-sum test), with NBS-HGG tumors from children less than three shown in blue and DIPGs from children less than 3 years of age shown in turquoise. Germline mutations are shown in red, with specific mutated genes labeled. Hypermutator samples were associated with germline PMS2 mutations and outlier mutation rates were also seen in samples with germline MSH6 or PMS2 mutation. In contrast, TP53 germline mutations show a range of mutation rates, including an outlier for low mutation rate. Germline $A T M$ mutation was associated with a lower mutation rate than the narrowest bulk. A tumor from a patient with germline NF1 mutation fell within the narrowest bulk. 
(Figure 2-5). In our cohort, chromothripsis resulted in oncogenic rearrangement, including $B T B D 1-N T R K 3$ fusion and rearrangement or amplification of

$P D G F R A$ and EGFR. Nearly half of all samples showing chromothripsis were collected before adjuvant therapy, indicating that the mechanism underlying their occurrence was, at least in some cases, independent of DNA-damaging therapeutics. SJHGG027_D, an NBS-HGG arising in a child with ataxia telangiectasia (A-T), had a relatively stable genome, despite a compromised DNA damage checkpoint due to the absence of functional ATM, ataxia telangiectasia mutated (Figures 2-5 and 2-6). Multiple subclones were identified in almost all HGG tumors. A founder clone or a descendant of a founder clone in the diagnosis tumor could seed the development of relapsed or autopsy tumor.

We identified TERT promoter mutations in only $2 \%$ of DIPGs and $3 \%$ of NBSHGGs, in strong contrast to the frequency of $86 \%$ identified in adult primary glioblastomas[129].

The genomic landscape of pediatric HGGs also includes frequent mutations in common cancer pathways, consistent with previous reports[50-52, 55, 56, 61, 63, 69]. TP53 mutations occurred in $42 \%$ of pediatric HGGs and were mutually exclusive with truncating mutations in the TP53-induced phosphatase PPM1D, previously shown to impair the TP53-dependent G1 cell cycle checkpoint (Figure 2-1)[130]. The G1 checkpoint regulators $C C N D 1, C C N D 2, C C N D 3, C D K 4$ and $C D K 6$ were predominantly amplified in DIPGs, whereas $C D K N 2 A$ homozygous deletion was restricted to NBSHGGs (Figure 2-1). Taken together, mutations affecting cell cycle regulation, including the TP53 and RB pathways, were found in 59\% of pediatric HGG (Figure 2-1).

This global view of the genetic landscape of pediatric HGG defines critical pathways driving a devastating spectrum of childhood brain tumors and identifies highfrequency mutations in potential therapeutic targets - ACVRl in DIPGs and NTRK fusions in NBS-HGGs in infants." 


\section{CHAPTER 3. FUSIONS INVOLVING THE NEUROTROPHIN FAMILY OF RECEPTOR TYROSINE KINASES IN GLIOMAGENESIS}

\section{Neurotrophin Signaling}

As mentioned previously, NTRK gene fusions appear to be oncogenic drivers in multiple glioma types, both in adult and pediatric HGGs as well as pLGGs[8, 107-109], and the genetic landscape of these tumors can differ markedly. pLGGs resemble infant NBS-HGGs in having quiet genomes whereas adult glioblastoma and non-infant pediatric HGGs exhibit higher mutation burdens[8-11, 107-109]. Such differences also extend into the prognosis of these gliomas, further emphasizing the heterogeneity among them. For adult and non-infant HGGs, the two-year survival rate is $<30 \%[36,40]$. By contrast, nearly $90 \%$ of children with LGG are alive a decade after diagnosis[131]. Although infants with NBS-HGG do fare better than non-infants, the clinical course of these patients is not akin to those with LGG[132], thereby debunking the idea that perhaps infant HGGs are diagnosed erroneously as high-grade or that they are merely low-grade tumors with high-grade histology.

The history of neurotrophin research is rich, dating back to the mid- $20^{\text {th }}$ century and includes the seminal work of Rita Levi-Montalcini and Stanley Cohen. In 1986, they were both awarded the Nobel Prize in Physiology and Medicine for the isolation of nerve growth factor (NGF) [133, 134]. In addition to NGF, the neurotrophin family of growth factors includes three other members: brain-derived neurotrophic factor (BDNF), neurotrophin-3 (NT-3), and neurotrophin-4 (NT-4). Collectively, these ligands and their cognate receptors (the Trk RTKs as well as the structurally unrelated p $75^{\mathrm{NTR}}$ ) are critical in the development of the nervous system. The consequences of neurotrophin signaling are diverse; mediating cell survival, differentiation, synaptic plasticity, and dendritic arborization as well as other cellular responses[135].

Neurotrophins are synthesized in the endoplasmic reticulum (ER) as precursors (proneurotrophins) with an N-terminal prodomain. Proteolytic cleavage of this domain either in the Golgi or secretory vesicles or extracellularly yields the mature ligand[136]. Existing as homodimers, mature neurotrophins can bind two different classes of cell surface receptors, the Trks or $\mathrm{p} 75^{\mathrm{NTR}}$. The former consists of three structurally related members (TrkA, TrkB, TrkC), while $\mathrm{p} 75^{\mathrm{NTR}}$ is part of the tumor necrosis factor receptor (TNFR) superfamily. While both receptor types can bind mature ligand, $\mathrm{p} 75^{\mathrm{NTR}}$ can additionally associate with proneurotrophins[137].

A common feature of the four neurotrophin gene loci is the existence of multiple 5 ' exons with the most 3' exon containing the open reading frame for the proneurotrophin. Alternative splicing to include or remove certain 5' exons leads to multiple mRNA transcripts, each with a distinct promoter. In fact, $B D N F$ mRNA has been shown to be regulated by eight different promoters. Further transcriptional control is achieved by virtue of the fact that all but one neurotrophin gene, $N G F$, contain alternative polyadenylation sequences. And yet an additional level of complexity is seen for $B D N F$; 
with a natural antisense transcript which can form a duplex with the coding strand, leading to RNA degradation. Although the role of these various transcripts remains to be seen, collectively they highlight the necessity of highly intricate gene expression for the development and maintenance of a functional nervous system[138].

Mature neurotrophins bind to Trk receptors. Alternative splicing of the sequence corresponding to the extracellular domains of all three NTRK genes alters the affinity each receptor has to the various ligands. Trk-neurotrophin coupling mediates receptor dimerization and subsequent trans-autophosphorylation of tyrosine residues. These phospho-tyrosines in turn activate three pathways associated with neurotrophins: the PI3K, MAPK, and phospholipase C gamma (PLC $\gamma$ ) networks (Figure 3-1). PI3K and MAPK signaling is initiated via the same residue whereas PLC $\gamma$ activation occurs through a different phospho-tyrosine. PI3K can bind directly to phospho-tyrosine by its p85 regulatory subunit or through an adaptor complex including Shc, Grb2, and Gab1. The catalytic portion of PI3K, p110, then converts phosphatidylinositol 4,5-bisphosphate (PIP2) to phosphatidylinositol $(3,4,5)$-trisphosphate (PIP3), with the product serving as a docking site for pleckstrin homology $(\mathrm{PH})$ domain-containing proteins, such as AKT. Full enzymatic activity of AKT is achieved through dual-phosphorylation at T308 and S473. At the same phospho-tyrosine responsible for PI3K activity, MAPK pathway signaling initiates with recruitment of Grb2 and SOS. A guanine nucleotide exchange factor, SOS then activates Ras and subsequently leads to downstream MAPK activation via phosphorylation of both threonine and tyrosine within a conserved TEY motif of the kinase domain. Together, both MAPK and PI3K converge on transducing signals for cell survival, proliferation, growth, and protein synthesis. A direct binding site for PLC $\gamma$ on Trk is generated when the most C-terminal tyrosine of the receptor is phosphorylated. Trk phosphorylation of PLC $\gamma-1$ Y 783 is both necessary and sufficient to induce enzyme activity. The expression of a second PLC $\gamma$ isozyme, PLC $\gamma-2$, is restricted to immune cells. Both PLC $\gamma$ and PI3K are similar in that they alter member lipid composition by acting on the same substrate. PLC $\gamma$ hydrolyzes PIP2 yielding the second messengers inositol trisphosphate (IP3) and diacylglycerol (DAG). Whereas the former facilitates $\mathrm{Ca}^{2+}$ release from the ER, DAG remains membrane-bound and promotes protein kinase $\mathrm{C}$ (PKC) signaling. Neurotrophin-Trk complexes are then internalized by clathrin-mediated endocytosis and can be recycled back to the membrane or degraded. Collectively, this process is known as canonical Trk signaling. Research has also identified two noncanonical Trk pathways: 1) neurotrophin-independent activation and 2) signaling via truncated isoforms lacking a kinase domain. However, the biological importance of these remains to be seen[139].

An enigma of sorts early during its study, $\mathrm{p} 75^{\mathrm{NTR}}$ is another type of neurotrophin receptor. Much of the confusion was initially due to the ability of $\mathrm{p} 75^{\mathrm{NTR}}$ to mediate both apoptotic and survival signaling. It has since been shown that these outcomes are highly context-dependent and rely on the receptor's ability to bind both proneurotrophins as well as mature ligand and also on its ability to form complexes either with Trk or with the type-1 receptor sortilin. Structurally, the intracellular domain lacks enzymatic activity and serves as a scaffold for adaptor recruitment[140]. 


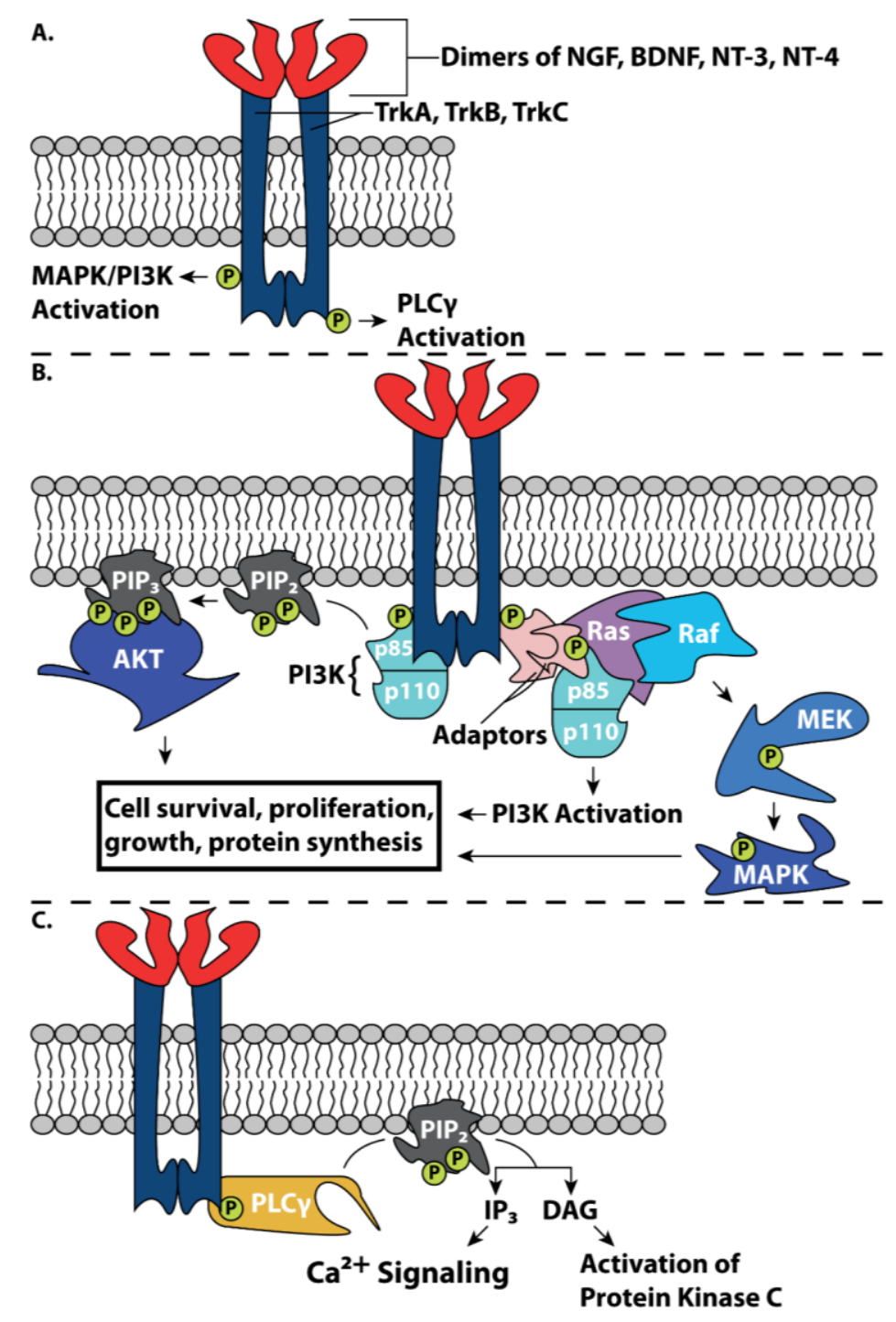

Figure 3-1. The NTRK signaling pathway. (A) Dimers of four ligands (NGF, BDNF, NT-3, NT-4) bind with varying affinity to the three NTRK receptors (TrkA, TrkB, TrkC). Upon neurotrophin binding, Trk receptors trans-phosphorylate at specific tyrosine residues. Phosphorylation of a C-terminal tyrosine leads to activation of PLC $\gamma$. When a more N-terminal tyrosine is phosphorylated, it results in activation of both MAPK and PI3K. (B) PI3K activation can occur through direct binding of $\mathrm{p} 85$, the regulatory subunit of PI3K, to Trk receptors. Alternatively, $\mathrm{p} 85$ can bind indirectly through its association with adaptor molecules and Ras. The catalytic p110 component of PI3K alters membrane lipid composition by converting PIP2 to PIP3, with the latter resulting in AKT activation. Ras can also initiate the MAPK signaling cascade. Both pathways transduce signals critical for cell survival, proliferation, growth, and protein synthesis. (C) Similarly to PI3K, PLC $\gamma$ alters membrane composition by acting on PIP2 to generate the second messengers IP3 and DAG. The former facilitates $\mathrm{Ca}^{2+}$ release from the ER whereas the latter induces the activity of PKC. 
Discovery of the first Trk receptor occurred when a novel gene fusion was identified in colorectal cancer. Genomic rearrangement combined the N-terminal sequence of TPM3, a tropomyosin gene, with a C-terminal sequence corresponding to the kinase domain of a previously unidentified RTK[141]. Named for its fusion partner, tropomyosin receptor kinase A, or TrkA as it would later be known, was the founding member of a family comprising two other such molecules. That initial report was published in 1986. Since then, additional NTRK fusion genes have been described across multitude cancer types (in both adult and childhood malignancies within and outside the CNS) with varying frequency (ranging from $0.2 \%-100 \%$ )[142]. And although those numbers may change as larger cohorts are studied and detection methods become increasingly sensitive, the fact remains that NTRK gene fusions drive tumorigenesis in numerous cancer contexts.

We have recently analyzed 127 patient samples, both DIPGs and NBS-HGGs, through whole-genome sequencing, whole-exome sequencing, and transcriptome sequencing. Our efforts revealed that $40 \%$ of infant NBS-HGGs contain NTRK fusion genes, and these were found in 7\% of pHGGs overall. When ranked by tier 1 mutation rate, infant tumors clustered together as having significantly quieter cancer genomes when compared to non-infants[8]. These findings, coupled with the observation that infants have a better prognosis than older children, suggests that infant NBS tumors are a distinct subgroup within $\mathrm{pHGG}[36,132]$. Although less frequent, NTRK fusion genes also occur in pLGG (nearly 4\%)[107, 108] and adult glioblastoma (1\%)[109]. Genomically, infant HGG resembles pLGG in exhibiting few mutations whereas adult glioblastomas appear

more somatically altered both in terms of copy number alterations and SNVs. Thus NTRK gene fusions are gliomagenic drivers throughout various development settings; yet it appears as if gliomas driven by the same oncogene can vary in tumor phenotype as a function of contextual differences. I hypothesize that the molecular heterogeneity of NTRK-driven gliomas stems from a dynamic vulnerability to oncogenic events inherent to developmental context.

\section{Methods}

\section{Implant studies}

Tumorigenesis implant studies were done as previously described[23]. Briefly, hGFAP-cre[143] and Trp53 $3^{\operatorname{lox} P / l o x P}[144]$ mice were crossed to drive homozygous Tp53 deletion. Primary astrocyte cultures were established from postnatal day 2 (P2) pups. Murine stem cell virus (MSCV) retroviral vectors were used to introduce expression of C-terminally FLAG-tagged NTRK fusions. The MSCV vector contained an IRESmCherry fluorescent reporter. Transduction efficiency was obtained by flow analysis to determine the percentage of cells expressing mCherry. Transduced astrocytes were subsequently implanted into the brain of athymic recipient mice. 


\section{Mouse dissection}

Animals requiring dissection were given $1 \mathrm{~mL}$ Avertin $(12.5 \mathrm{mg} / \mathrm{mL}) \mathrm{IP}$. Mice subsequently underwent thoracotomy and after puncture of the atria, were perfused with PBS. Harvested brain tissue was subsequently placed in 4\% PFA and whenever possible, additional snap frozen material was collected. Tails were collected to confirm initial genotyping results.

\section{Mouse modeling}

Mouse modeling was based on the Etv6-NTRK3 genetically engineered mouse model previously generated for the study of breast cancer in the lab of Dr. Stuart Orkin [114]. Briefly, the human NTRK3 cDNA sequence encoding the kinase domain was inserted into exon 6 of one of the murine Etv6 alleles. A lox-stop-lox cassette was placed immediately upstream from the cDNA sequence yielding a conditional knock-in allele. $T p 53^{\text {loxP/loxP }}$ mice[144] were also used to generate heterozygous and homozygous floxed alleles. Cre-mediated recombination was achieved using the transgenic nestin-cre ( $\mathrm{NCre}$ ) line, which utilizes enhancer and promoter elements of rat origin[145]. NCre has widespread expression of recombinase in the CNS beginning midway through embryogenesis (E10.5). We additionally utilized Rosa26-stop-EYFP mice as a reporter for Cre activity. In this line, the sequence encoding enhanced yellow fluorescent protein gene $(E Y F P)$ was inserted into the Rosa26 locus with an upstream lox-stop-lox sequence[146]. Expression of YFP will therefore be limited to cells in which Cre is active.

\section{Immunohistochemistry/Immunofluorescence}

Harvested brain tissue was placed in 4\% PFA in 1x PBS for 24h. Samples were then processed and embedded in paraffin. Sectioning of tissue was done at a thickness of 5 microns. Slides to be stained were deparaffinized and rehydrated. Slides were placed in citric acid $(\mathrm{pH}=6)$ and heated until boiling for antigen retrieval. Endogenous peroxidase was quenched by placing slides in $0.6 \% \mathrm{H}_{2} \mathrm{O}_{2}$ for 30 minutes. For blocking of nonspecific binding sites, slides were incubated in $10 \%$ goat serum in TBS-T $(0.01 \%$ Tween20 in TBS) for $1 \mathrm{~h}$. Afterwards, blocking solution was removed and primary antibody was diluted in $2 \%$ goat serum in TBS-T, with slides left to incubate overnight at $4^{\circ} \mathrm{C}$. For the second day of staining, slides were incubated with the species-appropriate biotinylated secondary antibody in $2 \%$ goat serum in TBS-T for $1 \mathrm{~h}$ at room temperature. Since secondary antibodies were biotinylated, signal amplification occurred though avidinbiotin complexing for $30 \mathrm{~m}$ at room temperature (Vector Laboratories, catalog number: PK-6100). NovaRed (Vector Laboratories, catalog number: SK-4800) and DAB (Vector Laboratories, catalog number: SK-4100) were the substrates utilized. Slides were then counter-stained, dehydrated, and coverslipped. Antibodies used: anti-FLAG (SigmaAldrich, F1804), anti-pPLC $\gamma 1$ T783 (Cell Signaling Technology, 14008), anti-pAKT S473 (Cell Signaling Technology, 9271), anti-p-p42/44 MAPK Thr202/Tyr204 (Cell 
Signaling Technology, 4370), anti-Ki67 (Novocastra, NCL-Ki67p), anti-Olig2 (Millipore, MABN50), and anti-Sox2 (Millipore, AB5603).

\section{Results}

NTRK fusion genes were found in $40 \%$ of infant NBS-HGG and in $7 \%$ of our overall pHGG cohort. Interestingly, one of the fusion genes, ETV6-NTRK3, has been described in other pediatric neoplasms such as congenital fibrosarcoma and congenital mesoblastic nephroma[147-149]. So to test the ability of ETV6-NTRK3 to drive glioma formation in vivo, we conducted an implantation study. Cortical astrocytes were harvested from $h G F A P$-cre; Tp $53^{\text {loxPloxP }} \mathrm{P} 2$ pups and primary cultures were established. Astrocytes were transduced with either an empty vector MSCV construct or one coding for ETV6-NTRK3, both with a mCherry fluorescent reporter. Transduction efficiency was $1.5 \%-2 \%$ for ETV6-NTRK 3 based on flow analysis. $2 \times 10^{6}$ cells were implanted into the cortex of CD-1 nude mice. Animals injected with astrocytes expressing ETV6-NTRK3 had a median survival of 36 days (Figure 3-2). The tumors from these mice were formally diagnosed as HGG and show evidence of MAPK and PLC $\gamma$ activity by IHC analysis. Fusion protein expression was confirmed by IHC detection of a C-terminally engineered FLAG tag (Figure 3-2).

In addition to our pHGG cohort, NTRK fusion genes have been found in $\mathrm{pLGG}$ and adult glioblastoma[107-109]; meaning the same driver promotes tumor formation in diverse contexts. This prompted us to examine how oncogenic potential changes as a function of developmental setting. To this end, we utilized genetically engineered mouse models. Subsequent experiments utilized three such models described in the "Methods" section of this chapter: Etv6-NTRK3 (EN3) knock-in, Nestin-cre (NCre), and Tp $53^{\text {loxP/loxP }}$ mice. We initially crossed heterozygous EN3 males to hemizygous $N C r e$ females to generate offspring that were positive for both alleles. Brainstem and cortical primary astrocyte cultures were established from P2 pups. A cell viability assay (XTT) was performed which showed an increase in cells expressing the fusion gene compared to controls (Figure 3-3). Expression of EN3 was confirmed by RT-PCR. Mice were bred to include Rosa26-stop-EYFP so that Cre activity could be determined. Over $90 \%$ of cells were YFP-positive by flow analysis.

In addition to mice with WT $T p 53$, we bred in $T p 53$ floxed alleles to generate NCre;EN3;Tp $53^{+/}$and NCre;EN3;Tp $53^{-/}$progeny. Tumor development was incompletely penetrant with WT $T p 53$, at least by gross examination. Five mice exhibited neurological symptoms and had tumor development that was apparent during dissection. Seven had to be euthanized due to unrelated health issues, such a paraphimosis or rectal prolapse, but one of these had evidence of tumor upon histological review. Seven mice were euthanized or died without neurological abnormality and did not have gross evidence of disease. Analysis of $N C r e$; EN3 mice remains ongoing and paraffinembedded tissue will need to be sectioned and evaluated microscopically. We were taken aback at the rate of tumor development in NCre;EN3;Tp $53^{-/-}$mice, with a median survival of 18 days (Figure 3-4). The median survival of $N C r e$;EN3; Tp $53^{+/-}$mice was 58 
A.
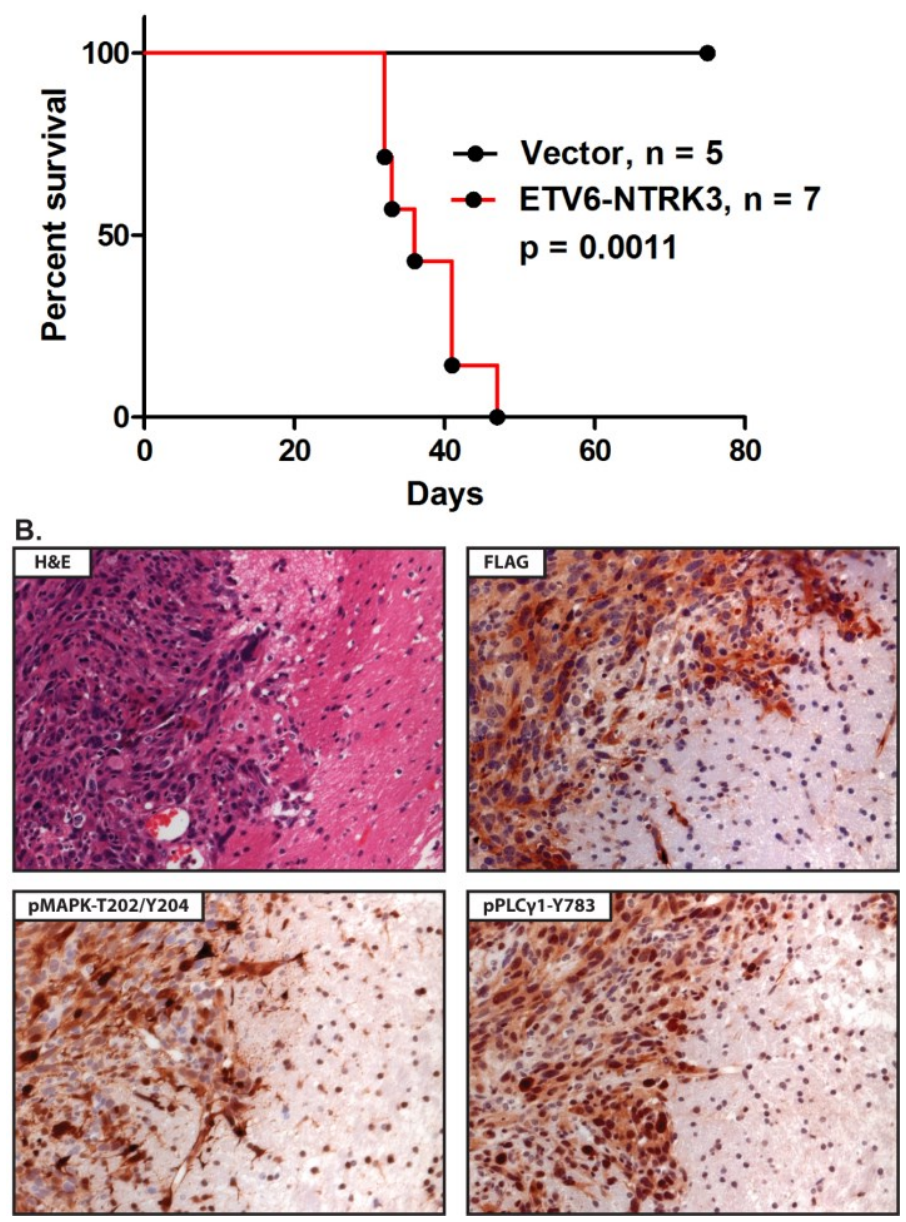

Figure 3-2. Astrocytes expressing ETV6-NTRK3 generate HGG when implanted into recipient mice.

Tp53-null astrocytes were transduced with retrovirus encoding ETV6-NTRK3 with a Cterminal FLAG tag. Cells were subsequently implanted into the cortex of

immunocompromised mice. (A) Kaplan-Meier comparing mice injected with astrocytes transduced with ETV6-NTRK3 or empty vector retrovirus. (B) ETV6-NTRK3 drives HGG formation with activation of MAPK and PLC $\gamma$. 20x H\&E and IHC sections using antibodies targeting the indicated epitopes. 


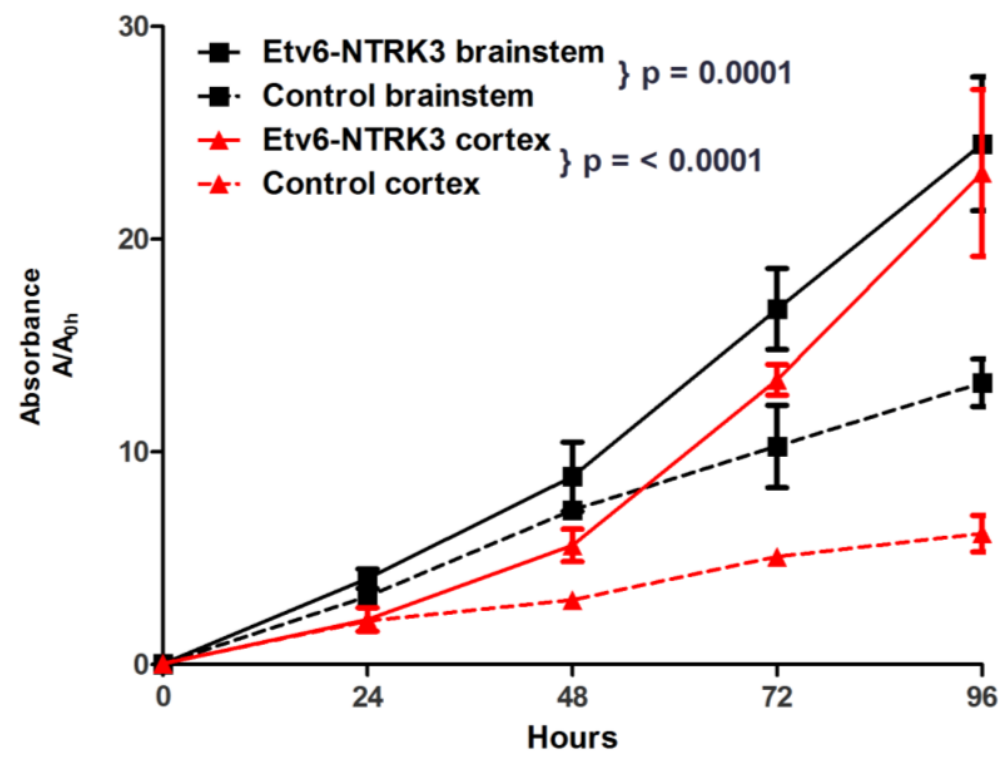

Figure 3-3. Expression of EN3 confers a proliferative advantage in vitro.

XTT from brainstem and cortical primary astrocytes harvested from $\mathrm{NCre}$ and $\mathrm{NCre}$;EN3 P2 pups. Findings consistent throughout all three replicates. 


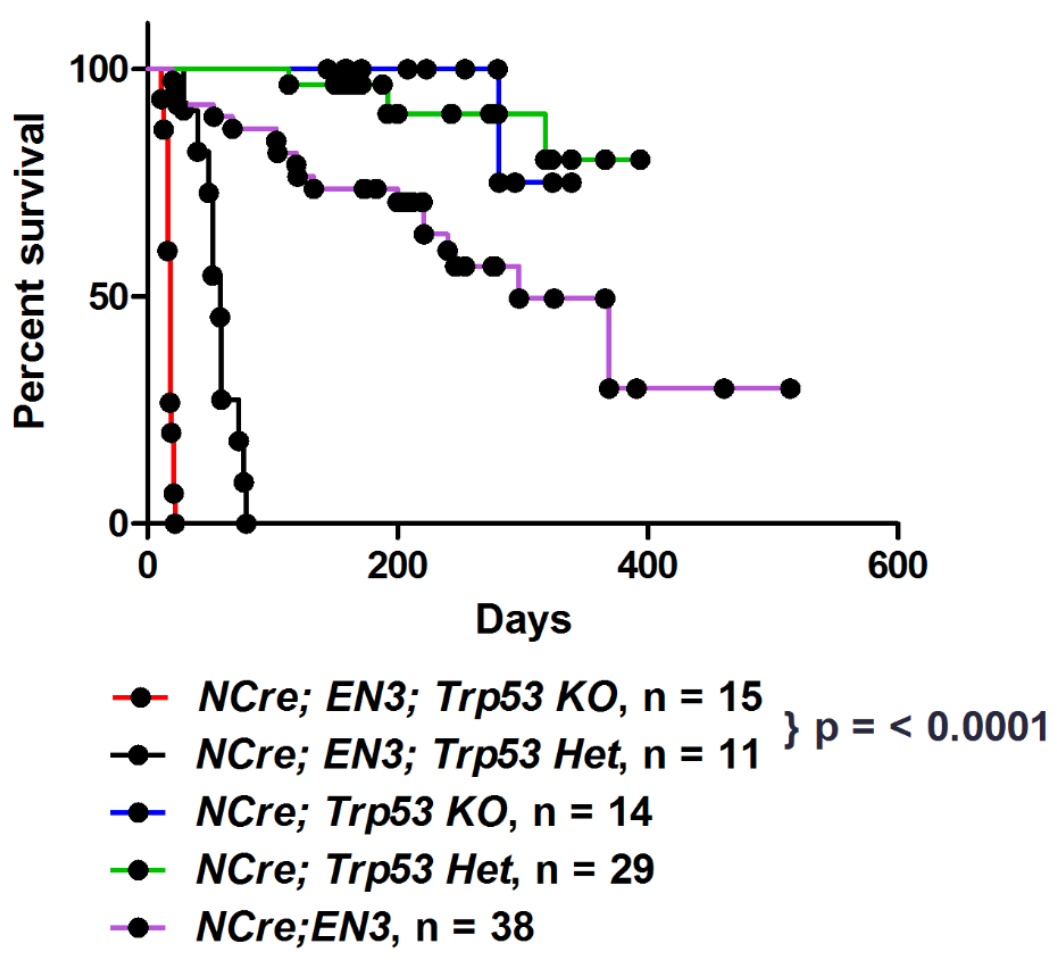

Figure 3-4. Kaplan-Meier curve of five cohorts: $N$ Cre $; E N 3 ;$ Trp $53^{-/-}$(red), NCre;EN3;Trp53 ${ }^{+/}$(black), NCre;EN3 (purple), NCre;Trp53-- (blue), and NCre;Trp $53^{+/-}$(green).

Five $N C r e ; E N 3$ mice had neurological symptoms with evident tumor during dissection. Seven $N C r e ; E N 3$ animals were euthanized due to unrelated health issues; one tumor upon histological review. Seven $N C r e ; E N 3$ mice were euthanized or died without neurological abnormality and did not have gross evidence of disease. Analysis of the NCre;EN3 cohort remains ongoing. 
days. Tumors in NCre;EN3; Tp53 $3^{-/-}$mice were diagnosed formally as HGG, exhibiting a high-proliferative index (by Ki-67), infiltration of normal parenchyma, and satellitosis

(Figure 3-5). Tumors were analyzed by IHC to detect pAKT-S473 and p-p42/44 MAPK Thr202/Tyr204, which is consistent with activation of PI3K and MAPK, respectively (Figure 3-6).

Given the rapid onset, we were interested to see earlier stages of tumorigenesis in NCre;EN3;Tp 53 $3^{--}$mice. We therefore examined the brains of P5 pups. Even by this time point there was high-grade disease (Figure 3-7). To see if EN3 was driving gliomagenesis in a tumor stem-cell-like population we performed immunofluorescence to detect Olig2 and Sox2. P5 tumors were largely comprised of discrete cell populations of either Olig2-positive or Sox2-positive cells. A very small minority of double-labeled cells were found and these appeared near tumor edges (Figure 3-7). As previously mentioned, NTRK signaling involves three pathways. In order to dissect the contribution each has on tumor formation, we needed to establish tumor cell lines. Tumorspheres were generated from three different $\mathrm{NCre} ; \mathrm{EN} 3 ; \mathrm{Tp} 53^{-/-} \mathrm{HGGs}$. To confirm that cultured cells were indeed tumor (and not immortalized NSCs given their Tp53-null status), we implanted 250,000 cells into the cortex of immunodeficient mice. All injected mice developed HGG (Figure 3-8), indicating successful establishment of in vitro renewable models of the NCre;EN3;Trp53 $3^{-/}$HGGs.

\section{Discussion}

To say ETV6-NTRK3 is a potent oncogenic driver would be an understatement. Despite a transduction efficiency of $1.5 \%-2 \%$, this fusion gene was able to generate HGGs with a shorter latency than similar experiments with the majority of cells expressing various PDGFRA mutants. But this was merely the beginning, as the mousehuman chimera of this same fusion protein was able to generate the first spontaneous pediatric model of HGG. This is far and away the most significant aspect of this work. For one, it provides a new platform for pre-clinical study. Our current approach to analyzing potential therapeutic agents includes in vivo testing using an implantation model. However, these experiments are in adult immunocompromised mice. The tumor latency of $\mathrm{NCre}$;EN3; Tp53 $3^{-/}$mice (median, 18 days) allows us to administer a compound to not only see if the agent works, but to also ascertain the toxicology profile during a physiologically relevant pediatric setting.

Our analysis continues. We collected snap frozen tissue and have since submitted this for RNASeq in the hopes of identifying the downstream targets that render this fusion gene so transforming. The EN3 knock-in model was initially developed for the study of breast cancer. In that context, researchers discovered that the fusion protein's oncogenicity was through activation of the AP1 transcriptional complex. AP1 itself is a dimeric complex comprised of members from the Jun, Fos, Maf, and ATF families. It will be interesting to see if EN3-driven gliomagenesis acts similarly or engages another downstream effector. Additionally, we will now look prenatally to uncover the initial 
A.

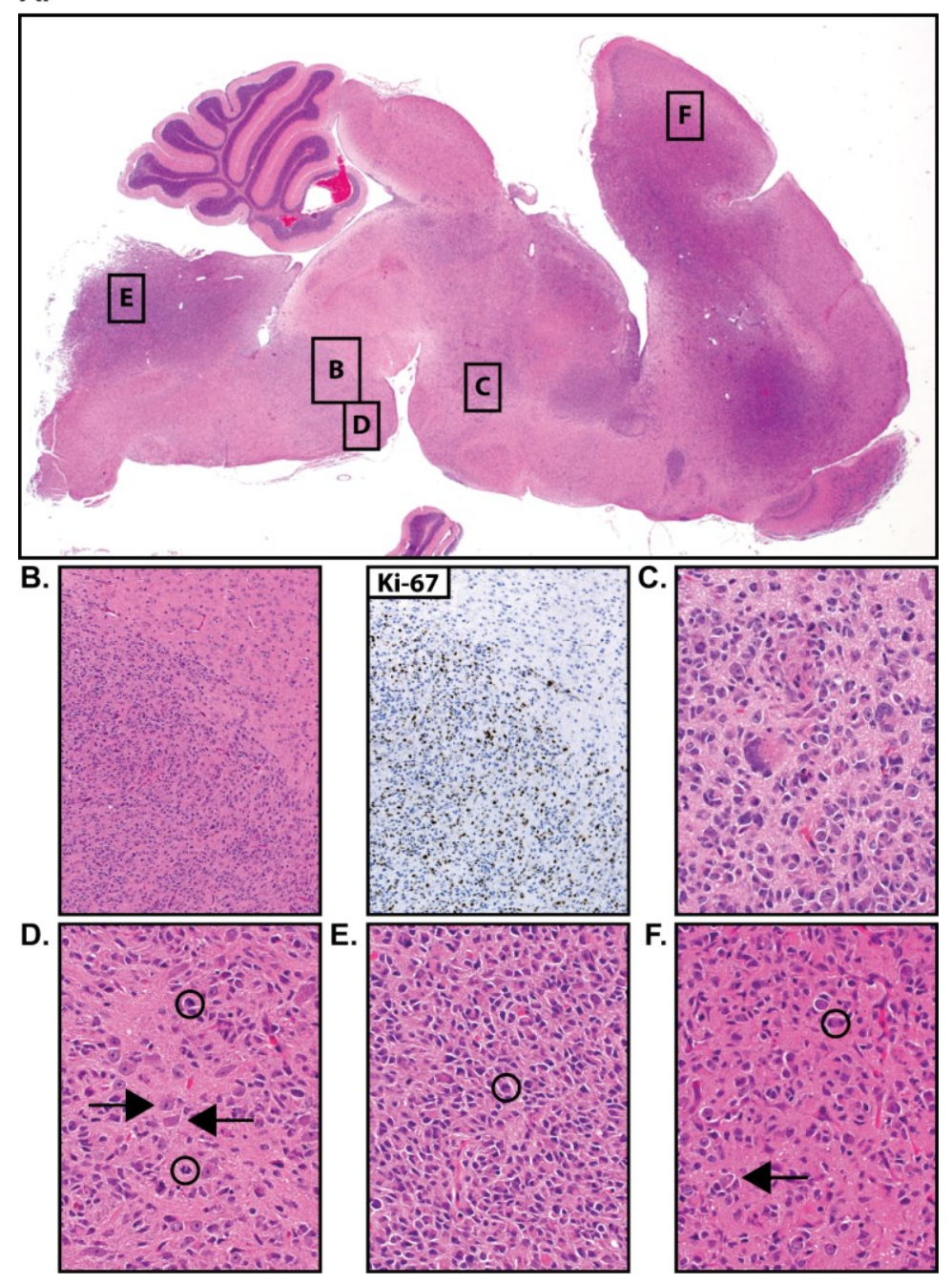

Figure 3-5. NCre;EN3;Trp53-- mice generate HGG.

(A) Sagittal H\&E section of a P18 tumor-bearing mouse showing extent of disease burden. (B) 10x H\&E and Ki-67 sections demonstrate a high proliferative index. (C-F) 40x H\&E. Native neurons are highlighted by arrows; mitotic figures are highlighted by circles. (C) Moderate to severe cytologic pleomorphism, including some multinucleated giant cells. (D) Infiltration of normal tissue by astrocytoma cells. (E) Mitotic figure. (F) Tumor cells demonstrate some satellitosis around cortical neurons and a mitotic figure. 

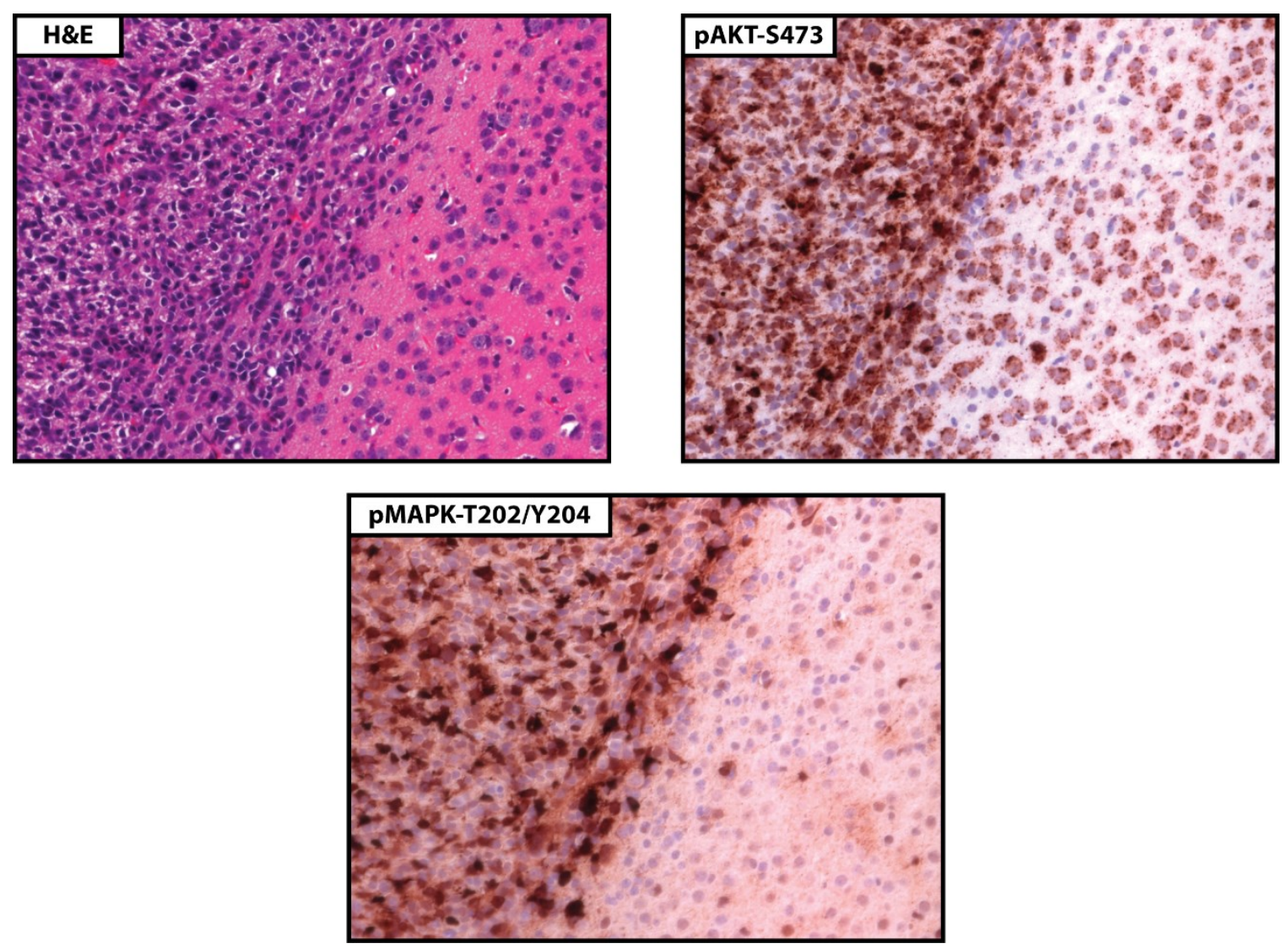

Figure 3-6. Tumors from NCre; EN3; Trp53-/- mice show activation of PI3K and MAPK.

20x H\&E and IHC sections using antibodies targeting the indicated epitopes. 
A.

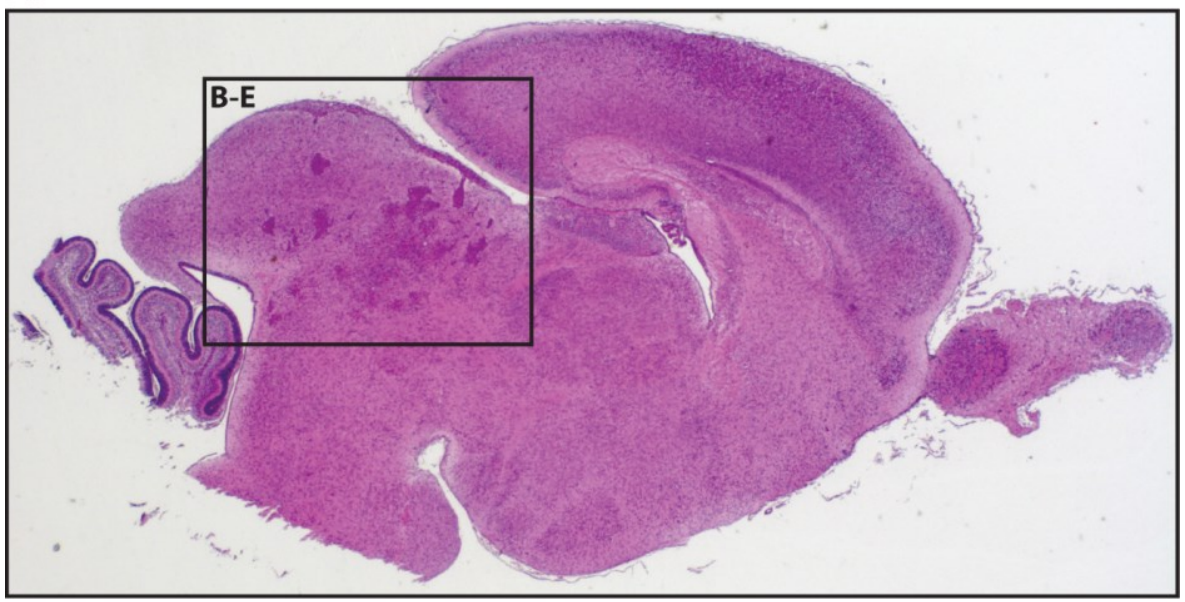

B.

C.

D.

E.

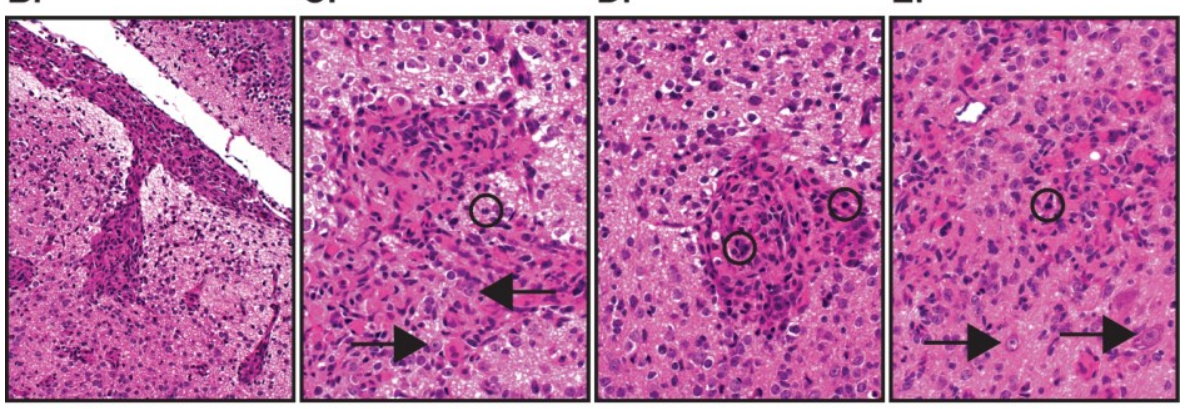

F.
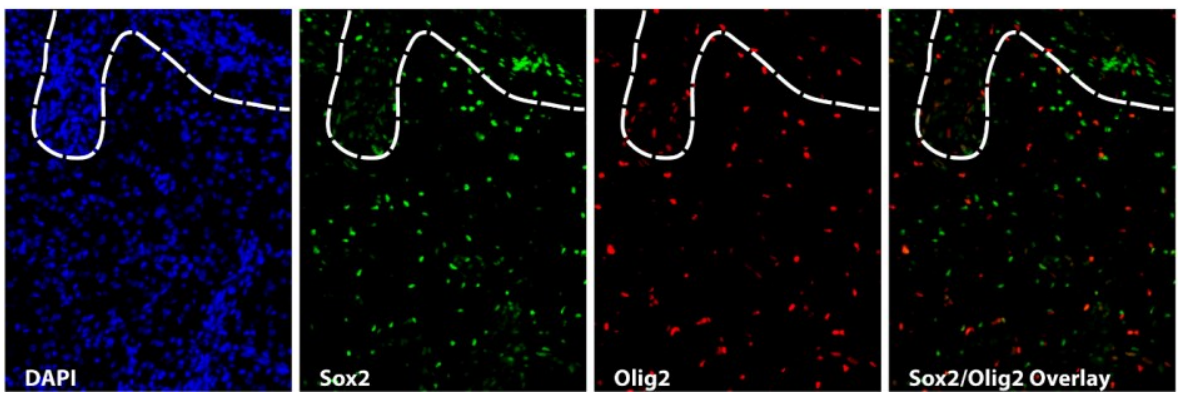

Figure 3-7. NCre;EN3;Trp53-/- mice have high-grade disease by P5.

(A) Sagittal H\&E section of a P5 mouse with evidence of HGG. (B) 20x H\&E section demonstrating sub-pial growth of infiltrating astrocytoma and involvement of the Virchow-Robin spaces. (C-E) 40x H\&E. Native neurons are highlighted by arrows; mitotic figures are highlighted by circles. (C, E) Sections demonstrating infiltrating astrocytoma with mitotic activity. (D) Section demonstrating infiltrating astrocytoma with perivascular growth pattern. (F) 20x immunofluorescence of tumor and adjacent normal with triple labeling of DAPI, Sox2, and Olig2. Image taken to show tumor involvement of Virchow-Robin space. Tumor consists largely of two discrete populations: singly labeled Olig2 or Sox 2 positive cells. The few double positive cells appear in tumor edges. 


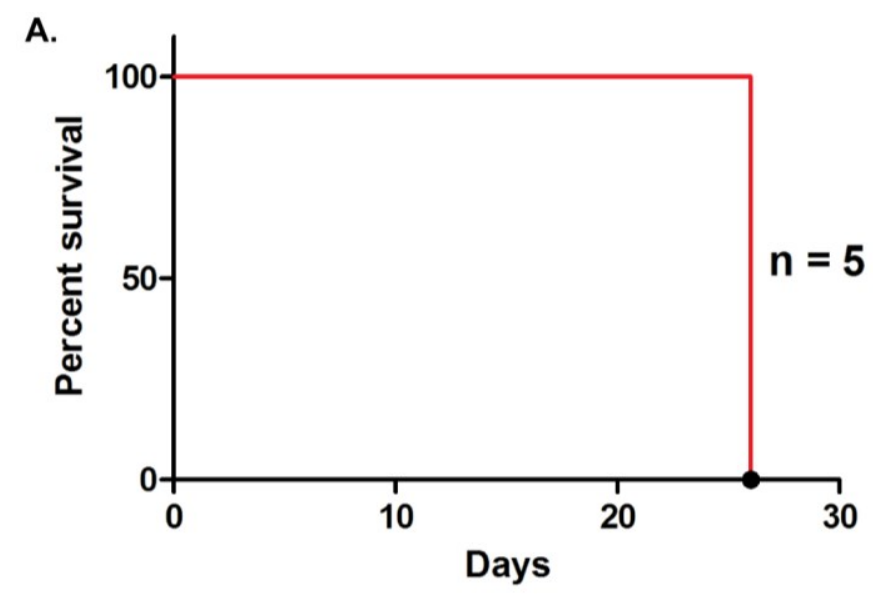

B.
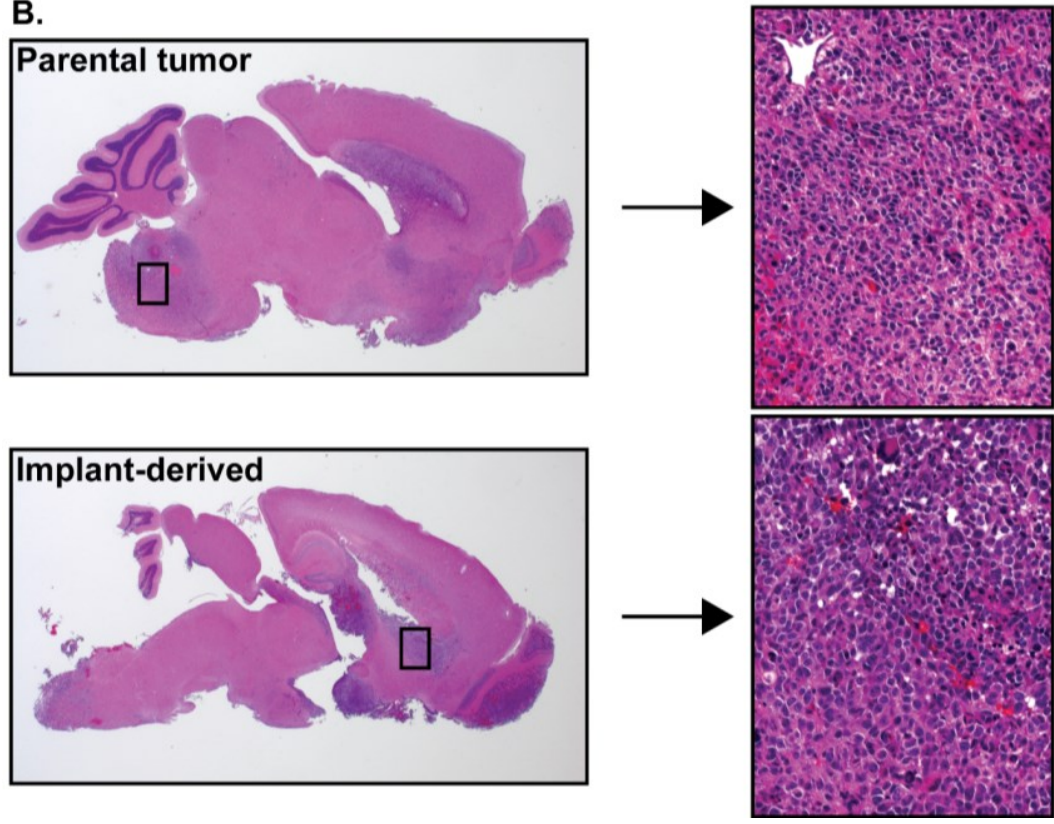

Figure 3-8. Tumorspheres from NCre; EN3; Trp53-/- mice generate HGGs when implantated into recipient mice.

Tumorsphere cell lines were established from a NCre;EN3; Trp $53^{-/-}$tumor-bearing mouse that was euthanized at P21. At passage 2, 250,000 cells were injected into the cortex of immunocompromised mice. (A) All recipient mice were euthanized 26 days postinjection due to moribund status. (B) Sagittal H\&E sections demonstrate that implantderived HGGs recapitulate features of the parental spontaneous tumor. 
stages of tumorigenesis; to see how expression of ETV6-NTRK3 affects native cell populations during the beginning of the gliogenic window.

We are hopeful this model and these studies will yield insight into how these tumors develop. Currently in the pHGG research community, phrases such as "unique selective pressures" and "developmental context" form the backbone of our manuscript introductions and discussions. They are essentially fancy descriptors of the black box that is HGG formation in children. Perhaps now we can start to shed some light on this process, giving our words a bit more meaning. 


\section{CHAPTER 4. DISCUSSION}

\section{ACVR1}

ACVR1 mutations are somatic heterozygous missense alterations, with the remaining WT allele intact. Even without considering any functional data, this pattern is suggestive of gain-of-function; making mutant ACVR1 an oncogene.

The identification of $A C V R 1$ mutations in DIPG was similar to the discovery of histone mutations in that they both implicated novel biology in disease pathogenesis. Although showing dysregulation in the 3 core pathways of HGG, prior genetic and molecular studies had never found a role for BMP signaling.

BMPs belong to the transforming growth factor beta (TGF $\beta$ ) superfamily along with activins. They bind membrane receptors known as type II receptors. Two type II receptors then bind two type I receptors, which ACVR1 is, forming a tetrameric complex. When this occurs, type II receptors phosphorylate ACVR1 in a GS rich regulatory domain. In the unstimulated state, this region forms a docking site for FKBP12, rendering the kinase of the type I receptor inactive. Phosphorylation of this GS domain causes FKBP12 to dissociate, allowing ACVR1 to phosphorylate its targets, the receptorregulated SMADs 1/5/8 (R-SMADs). A trimer is then formed consisting of two RSMADs and common-mediator SMAD4 (co-SMAD). This trimer subsequently translocates to the nucleus and mediates expression of downstream targets including the inhibitor of DNA-binding/differentiation (Id) family of transcription factors. Noncanonical BMP signaling is SMAD independent and leads to activation of p38 MAPK[150].

As previously mentioned, our group and others have shown that $A C V R 1$ mutations render its kinase domain constitutively active. Recently, two publications have uncovered an additional function: the ability of mutant ACVR1 to signal upon binding of a traditional BMP antagonist, activin A[151,152]. Normally, activin A transduces signals along the TGF $\beta$ axis while simultaneously preventing BMP activation by binding ACVR2A and ACVR2B, the type II receptors known to couple with ACVR1[150]. As a result, when mutant ACVR1 is present, the pathway will be active whether or not ligand is present and when inhibitor is present as well.

The p.K27 position of histone H3.1 and H3.3 can undergo mono-, di-, and trimethylation as well as acetylation, and these modifications are carried out by a number of enzymes[90, 91, 120]. p.K27M mutations lead to a global loss of histone H3 K27 trimethylation, despite the mutant protein being a minority of the total cellular fraction of histone H3[116-118]. After sequencing of our cohort, we were surprised to learn that the evolutionary pressure in $\mathrm{pHGG}$ is specific for selecting p.K27M to reduce trimethylation, to the exclusion of possible alterations to the modification machinery affecting that amino acid[8]. Similarly, the tumor dynamics within DIPG target the BMP pathway solely through mutant ACVR1, conferring a selective advantage specific to the 
brainstem given these mutations are restricted to this area[8-11]. It is for these reasons, in addition to frequency, that both these alterations are appealing therapeutic targets. These tumors have selected very specific paths for achieving particular functional outcomes, with no evidence of alternative mechanisms. That is not to say one would not emerge in the face of targeted therapy. In fact, that is to be expected given how aggressive these cancers are. For RTKs, resistant populations are already present even prior to any proposed targeted agent; we see the same tumor targeting multiple RTKs either in the same cell or in different subclones[51]. However, it seems that resistance would have to be acquired in the case of ACVR1 and histone H3. Even if only transiently effective, such treatments may add a month or two months to overall survival, which are meaningful steps we need to take in order to reach our goal of a cure.

In individuals with FOP, mesenchymal stem cells aberrantly differentiate to osteoblasts[95]. Knowing activin A mediates BMP signaling through mutant ACVR1 helps us to understand why heterotopic ossification is exacerbated by tissue injury. Activin $\mathrm{A}$ is a known mediator of inflammation and works in antagonizing the BMP pathway. However, mutant ACVR1's capacity to phosphorylate R-SMADs upon activin A binding transforms an environment that typically restricts the expansion of boneforming cells into one that promotes it. So is there an inflammatory component to HGGs? Certainly; microglia can be seen in and around tumors and a hallmark of grade IV tumors is necrosis. But these features are shared by all HGGs, regardless of age and location. It is worth mentioning that we found BMP activation regardless of $A C V R l$ status, with no appreciable difference in levels of phospho-SMAD 1/5 between mutant and WT tumors[8]. Perhaps then all pHGGs rely on BMP signaling. More pronounced inflammation may exist in certain tumors, initially suppressing pathway activity. But once $A C V R 1$ is mutated, the inflammatory milieu switches to promote BMP signaling. This possibility is supported by the fact that an inflammatory expression signature significantly associates with $A C V R 1$ mutation[8].

Can restriction of $A C V R I$ mutation to the brainstem provide insight to a potential cell of origin? BMP activation in NSCs promotes astrogliogenesis and prevents oligodendrocyte differentiation[153]. Given no increase in cancer incidence in FOP patients, we believe ACVR1 is playing a role in tumor progression rather than initiation. Imagine then a scenario where a tumor in the brainstem is growing, causing local inflammation. The microenvironment is now rich in cytokines and other molecules, including activin A. A hypothetical cancer stem cell, a transformed NSC, acquires an $A C V R 1$ mutation that, rather than promoting terminal differentiation, leads to unchecked proliferation of a tumor transit amplifying astrocytic population. Several cell types have demonstrated gliomagenic potential, but perhaps the trajectory outlined above occurs in a subset of DIPGs, given the enrichment of $A C V R 1$ mutations in younger patients. The pons itself is broken down into a ventral basis pontis and dorsal tegmentum, and DIPGs are thought to arise from the former. So what is in this area of the pons? Longitudinal and transverse fibers in addition to the pontine nuclei. There exists a Nestin+ cell population in the human ventral pons that is most prevalent shortly after birth. Interestingly, this cell type makes a resurgence in middle childhood, roughly corresponding to the ages of highest incidence for DIPG[154]. 


\section{NTRK Gene Fusions and Infant NBS-HGG}

To say we were taken aback at the oncogenic capacity of NTRK fusion genes would be an understatement. Despite an abysmal transduction efficiency of less than $2 \%$, Tp53-null astrocytes transduced with an ETV6-NTRK3 retroviral construct managed to generate HGGs within a few weeks after implantation. And in our spontaneous model, when the mouse-human chimera of this same alteration is expressed with concomitant Tp53 deletion in embryonic NSCs, it was able to establish true pediatric disease.

NTRK signaling involves three pathways: MAPK, PI3K, and PLC $\gamma$. Early investigations looking at NTRK focused on the contribution of the first two and understandably so, given their involvement in cancer. Site-directed mutagenesis of the tyrosine responsible for PLC $\gamma$ activation did not abolish the ability of fusion genes to transform NIH3T3 cells[155], an immortalized line of mouse fibroblasts. However, that is not to say the pathway is not contributing. We see evidence that it is active in our systems, and part of the potency of NTRK fusion genes may very well stem from this axis. PLC $\gamma$ converts PIP2 to the second messengers IP3 and DAG. The former is responsible for $\mathrm{Ca}^{2+}$ release from the ER while DAG remains membrane-bound and activates PKC[139]. The list of downstream targets of the various $\mathrm{PKC}$ isozymes is extensive. Yet those isozymes found to promote tumor formation have been linked to activation of both PI3K and MAPK, in addition to upregulation of cyclin D leading to $\mathrm{pRb}$ hyperphosphorylation[156]. Prolonged NTRK signaling leads to downregulation of its associated receptors, including at the level of expression[139]. These fusion genes are impervious to this type of regulation however as they are under the control of their Nterminal partner; which, in addition to the activation of three pathways, could contribute to their oncogenic potential. We were intrigued to see that the NTRK fusion genes in our cohort were not highly expressed as is the case with most other oncogenes[8]. Perhaps this diminished susceptibility to transcriptional control circumvents the need for increased expression to overcome potential inhibition.

Interestingly, the frequency of TP53 mutation is significantly less in infant NBSHGGs $(10 \%)$ than it is in pHGG overall $(55-70 \%)[7,8]$. Although we were able to generate a few tumors without deletion of the gene, NTRK-driven gliomagenesis required knockout of Tp53. The infant developmental context may require targeting of the pathway by other means, such as upregulation of the p53 negative regulator MDM2 or downregulation of the CDK inhibitor p21, itself a p53 effector. Given how barren these tumors are mutationally, dysregulation of the aforementioned targets, or any others, may occur at the epigenetic level rather than through genetic alteration. Then again, this particular developmental setting may not require direct perturbation.

Acute lymphoblastic leukemia (ALL) is the most common pediatric malignancy. And like pHGG, a small subset of these occur in very young children. Known as infant ALL, these cancers typically arise within the first year of life. Similar to the enrichment of NTRK fusion genes in infant NBS-HGG, infant ALL exhibits characteristic rearrangements involving the mixed lineage leukemia (MLL) gene in a majority of cases 
$(\sim 80 \%)[157]$. Another similarity between these two diseases is the paucity of somatic mutations in their cancer genomes. But unlike the youngest pHGG patients who have a better prognosis than older children, infants with ALL appear to have a particularly aggressive disease and fare worse than non-infants. So what could account for this? MLL is a methyltransferase responsible for histone post-translation modification, making it a global transcriptional regulator. It would therefore be reasonable to suspect that rearrangements leading to MLL fusions would involve its enzymatic SET domain, but this is not the case. These gene fusions result in epigenetic deregulation and disrupt transcription of target genes[157]. Any mutation that is selected for enhances evolutionary fitness. And while the same could be said for those leading to epigenetic dysregulation, there is a distinction between these alterations and others: widespread epigenetic deregulation perturbs numerous genes rather than just one. In infant ALL we see this whereas in infant NBS-HGG we do not, and this could potentially explain the difference in prognosis between the two.

NTRK fusion genes drive HGG formation in a number of developmental settings: in NBS tumors both in infants and older children, DIPG, and adult glioblastoma, which arise predominantly in the cortex [8, 107-109]. A still unresolved issue in HGG biology is the identification of a putative cell of origin. In the previous section of this discussion I explored this subject as it pertains to DIPG. To summarize briefly, brainstem HGGs are thought to arise from the ventral pons. A nestin-expressing precursor cell exists in this area and is found to be at its highest numbers shortly after birth, slowly dwindling until about 2 years of age. This population subsequently increases around the same time that children typically are diagnosed with DIPG[154]. For adults, a legitimate case can be made for NSCs, OPCs, and astrocytic progenitors as these are actively proliferating. Various HGG models have been developed targeting genetic alterations in these cell types[158]. Others might be tempted to include neurons as a potential cell of origin, but arriving at such a conclusion would require a bit of mental gymnastics. Neurons are postmitotic, meaning that to be a HGG cell of origin, all necessary hits would have to occur in the same cell or robustly induce proliferation of a post-mitotic neuron to increase the likelihood of acquiring co-mutation of other critical genes. Possible? Yes, but unlikely.

The issue is a bit more complex and nuanced when we look at infant and noninfant HGGs outside the brainstem. In mice, gliogenesis begins by E15 and is largely complete by the first month of postnatal life. The pediatric brain is therefore abundant with mitotically active NSCs, OPCs, and astrocytic progenitors relative to the adult brain[17]. Our experimental systems demonstrate that P2 astrocytes, through implantation, and embryonically targeted NSCs have the capacity to generate HGGs. However, we must keep in mind that although genetic alterations are targeted in a specific cell type, it may be their more differentiated progeny that give rise to a tumor[159]. Aside from age, there are two key differences between infants and noninfants with NBS-HGG that lead us to believe that they originate from different cell types: 1) the difference in prognosis and 2) the difference in mutation burden. Infants fare better than non-infants[132] and also have significantly quieter cancer genomes than older children[8]. So what then could account for this? In their "Hallmarks of Cancer," Robert Weinberg and Douglas Hanahan detail the attributes a cell gains in the 
progression toward malignancy[160,161]. To a certain extent, stem cells and cancer cells resemble one another. Meaning that on the path leading to cancer, stem cells have a head start so to speak and possess a number of these traits intrinsically. Maybe this ancestral cell is what gives rise to infant tumors; its quiet genome a reflection of the minimal steps needed to get disease. Maybe the cell of origin for older children falls more along the differentiated side of the spectrum; with its mutation burden a reflection of the need to acquire more requisite traits of transformation which it was originally lacking. This remains an active area of investigation. In addition to using a constitutively active Cre, we can use inducible forms, under the same and different promoters, to introduce Etv6NTRK3 in various cell types and ages to see: 1) how these alter a cell's ability to be transformed, 2) if the difference is due to a change in the transcriptional program, 3) if the NTRK-associated pathways are equivalently contributing to that program, 4) if the program is unique to NTRK fusion genes or shared by RTKs in general (comparing to a different model in the lab using transgenic mutant PDGFRA), and 5) if the responsible pathways can be targeted therapeutically. We are excited about this model for a number of reasons. First and foremost, it provides us a unique platform to perform preclinical testing. No other system yet described, be it implantation or spontaneous, generates HGG in a pediatric timeframe. This minimal latency will also enable us to work backwards from endpoint disease, similar to our P5 analysis, to see just how the fusion gene is functioning and in which cell type, giving us an opportunity to see the multistep tumorigenesis process in vivo. Tumorsphere cell lines can be established as well. By treating these with inhibitors of the NTRK-associated pathways it will be possible to determine if a particular axis is responsible for the fusion gene's transcriptional program, thereby revealing a potential therapeutic vulnerability.

A rather puzzling topic is that of NTRK fusion-driven infant NBS-HGG and pLGG. Their histologies differ and so too do their prognoses, but they resemble one another in that they are both minimally somatically altered. As previously mentioned, the prognosis for infant NBS-HGG lies in-between that of older children with HGG and pLGG. So what are the features of pLGGs harboring NTRK fusions? First, it should be noted that we are talking about four cases discovered thus far[107, 108]. Actually, in our cohort of pHGG, we had 10 infant tumors and four of those had NTRK gene fusions[8]. So between the two, we are talking about 8 cases total. But the four pLGGs that have been identified are in children older than 3 years and with tumors in the cortex $[107,108]$. Now for the hand-waving. One could see this age discrepancy and conclude that these tumors arise from a different cell of origin or from the same cell but in a different time point in development. But LGGs are, by definition, slow-growing. Let us entertain the idea that both these tumors started from the same cell type. So how then do we account for their differences? Perhaps those that progressed to high-grade acquired additional hits but at the epigenetic level. Our spontaneous model indicates that NTRK-driven tumorigenesis requires few cooperating events. And so we could literally be talking about an alteration or two being the difference in this very specific instance. 


\section{Closing Remarks}

I was extremely fortunate to be a graduate student during a period of tremendous growth in our understanding of the pHGG landscape. In the last 4 plus years the power of unbiased interrogation via next-generation sequencing has given researchers the ability to peer into the genetics of these tumors at an unprecedented level. The result? The suspicions of yesterday are now the truths of today: pediatric and adult HGGs are related but distinct. And when we take a closer look at children with HGG, we begin to appreciate that even within this age group, there is a spectrum of disease. Our work and the work of others identifies previously undescribed oncogenic drivers in different subgroups of pHGG: histone p.K27M and $A C V R 1$ mutations in DIPG, less frequent p.K27M and FGFR1 mutations in thalamic tumors, $H 3 F 3 A$ p.G34R/V alterations in laterally-located cortical HGGs, and the enrichment of NTRK fusion genes in infant NBS-HGG. This spatiotemporal distribution of genetic drivers underscores the importance and the uniqueness of the selective pressures involved in pediatric gliomagenesis.

This process, the doctoral training process, can be a whirlwind sometimes; empowering you one day only to leave you exasperated the next. And this is a good thing. Feeling that happiness and frustration is affirmation that you care about what you do. As I transition back to medical school I do so with a sense of excitement; not only for my own personal sake but also because I do so in a new era of pHGG study. The community has done well in identifying the genetic events that drive these tumors. And although we can take pride in those efforts, our satisfaction is short-lived. The imperative now is to use this information to shape the next generation of clinical trials. So imagine the position I find myself in: to have contributed in the research that leads to the trials I may be involved with as a physician-scientist. I am humbly grateful, now and always. Thank you. 


\section{LIST OF REFERENCES}

1. Mukherjee, S., The Emperor of All Maladies: A Biography of Cancer. 2010, New York: Simon \& Schuster.

2. Dahm, R., Friedrich Miescher and the discovery of DNA. Dev Biol, 2005. 278(2): p. 274-88.

3. Vogt, P.K., Retroviral oncogenes: a historical primer. Nat Rev Cancer, 2012. 12(9): p. 639-48.

4. Dishop, M.K. and S. Kuruvilla, Primary and metastatic lung tumors in the pediatric population: a review and 25-year experience at a large children's hospital. Arch Pathol Lab Med, 2008. 132(7): p. 1079-103.

5. Zhang, J., et al., Germline Mutations in Predisposition Genes in Pediatric Cancer. N Engl J Med, 2015. 373(24): p. 2336-46.

6. Vogelstein, B., et al., Cancer genome landscapes. Science, 2013. 339(6127): p. 1546-58.

7. Jones, C. and S.J. Baker, Unique genetic and epigenetic mechanisms driving paediatric diffuse high-grade glioma. Nat Rev Cancer, 2014. 14(10).

8. Wu, G., et al., The genomic landscape of diffuse intrinsic pontine glioma and pediatric non-brainstem high-grade glioma. Nat Genet, 2014. 46(5): p. 444-50.

9. Buczkowicz, P., et al., Genomic analysis of diffuse intrinsic pontine gliomas identifies three molecular subgroups and recurrent activating ACVR1 mutations. Nat Genet, 2014. 46(5): p. 451-6.

10. Fontebasso, A.M., et al., Recurrent somatic mutations in ACVR1 in pediatric midline high-grade astrocytoma. Nat Genet, 2014. 46(5): p. 462-6.

11. Taylor, K.R., et al., Recurrent activating ACVR1 mutations in diffuse intrinsic pontine glioma. Nat Genet, 2014. 46(5): p. 457-61.

12. Ostrom, Q.T., et al., CBTRUS Statistical Report: Primary Brain and Central Nervous System Tumors Diagnosed in the United States in 2008-2012. Neuro Oncol, 2015. 17 Suppl 4: p. iv1-iv62.

13. Voltz, R. and G.D. Borasio, Palliative therapy in the terminal stage of neurological disease. J Neurol, 1997. 244 Suppl 4: p. S2-10.

14. Taillibert, S., F. Laigle-Donadey, and M. Sanson, Palliative care in patients with primary brain tumors. Curr Opin Oncol, 2004. 16(6): p. 587-92.

15. Deeken, J.F. and W. Loscher, The blood-brain barrier and cancer: transporters, treatment, and Trojan horses. Clin Cancer Res, 2007. 13(6): p. 1663-74.

16. Louis, D.N., et al., The 2007 WHO classification of tumours of the central nervous system. Acta Neuropathol, 2007. 114(2): p. 97-109.

17. Gallo, V. and B. Deneen, Glial development: the crossroads of regeneration and repair in the CNS. Neuron, 2014. 83(2): p. 283-308.

18. Holland, E.C., et al., Combined activation of Ras and Akt in neural progenitors induces glioblastoma formation in mice. Nat Genet, 2000. 25(1): p. 55-7.

19. Alcantara Llaguno, S., et al., Malignant astrocytomas originate from neural stem/progenitor cells in a somatic tumor suppressor mouse model. Cancer Cell, 2009. 15(1): p. 45-56. 
20. Jacques, T.S., et al., Combinations of genetic mutations in the adult neural stem cell compartment determine brain tumour phenotypes. EMBO J, 2010. 29(1): p. 222-35.

21. Chen, J., et al., A restricted cell population propagates glioblastoma growth after chemotherapy. Nature, 2012. 488(7412): p. 522-6.

22. Bachoo, R.M., et al., Epidermal growth factor receptor and Ink4a/Arf: convergent mechanisms governing terminal differentiation and transformation along the neural stem cell to astrocyte axis. Cancer Cell, 2002. 1(3): p. 269-77.

23. Endersby, R., et al., Nonredundant functions for Akt isoforms in astrocyte growth and gliomagenesis in an orthotopic transplantation model. Cancer Res, 2011. 71(12): p. 4106-16.

24. Paugh, B.S., et al., Novel oncogenic PDGFRA mutations in pediatric high-grade gliomas. Cancer Res, 2013. 73(20): p. 6219-29.

25. Liu, K.W., et al., SHP-2/PTPN11 mediates gliomagenesis driven by PDGFRA and INK4A/ARF aberrations in mice and humans. J Clin Invest, 2011. 121(3): p. 905-17.

26. Ghazi, S.O., et al., Cell of origin determines tumor phenotype in an oncogenic Ras/p53 knockout transgenic model of high-grade glioma. J Neuropathol Exp Neurol, 2012. 71(8): p. 729-40.

27. Munoz, D.M., et al., Differential transformation capacity of neuro-glial progenitors during development. Proc Natl Acad Sci U S A, 2013. 110(35): p. 14378-83.

28. Munoz, D.M., et al., Loss of 53 cooperates with K-ras activation to induce glioma formation in a region-independent manner. Glia, 2013. 61(11): p. 186272.

29. Radke, J., G. Bortolussi, and A. Pagenstecher, Akt and c-Myc induce stem-cell markers in mature primary p53(-)/(-) astrocytes and render these cells gliomagenic in the brain of immunocompetent mice. PLoS One, 2013. 8(2): p. e56691.

30. Chow, L.M., et al., Cooperativity within and among Pten, $p 53$, and Rb pathways induces high-grade astrocytoma in adult brain. Cancer Cell, 2011. 19(3): p. 30516.

31. Zong, H., L.F. Parada, and S.J. Baker, Cell of origin for malignant gliomas and its implication in therapeutic development. Cold Spring Harb Perspect Biol, 2015. 7(5).

32. Verhaak, R.G., et al., Integrated genomic analysis identifies clinically relevant subtypes of glioblastoma characterized by abnormalities in PDGFRA, IDH1, EGFR, and NF1. Cancer Cell, 2010. 17(1): p. 98-110.

33. Dawson, M.R., et al., NG2-expressing glial progenitor cells: an abundant and widespread population of cycling cells in the adult rat CNS. Mol Cell Neurosci, 2003. 24(2): p. 476-88.

34. Dimou, L., et al., Progeny of Olig2-expressing progenitors in the gray and white matter of the adult mouse cerebral cortex. J Neurosci, 2008. 28(41): p. 10434-42.

35. Geha, S., et al., NG2+/Olig2+ cells are the major cycle-related cell population of the adult human normal brain. Brain Pathol, 2010. 20(2): p. 399-411. 
36. Broniscer, A. and A. Gajjar, Supratentorial high-grade astrocytoma and diffuse brainstem glioma: two challenges for the pediatric oncologist. Oncologist, 2004. 9(2): p. 197-206.

37. Broniscer, A., et al., Clinical and molecular characteristics of malignant transformation of low-grade glioma in children. J Clin Oncol, 2007. 25(6): p. 682-9.

38. Chassot, A., et al., Radiotherapy with concurrent and adjuvant temozolomide in children with newly diagnosed diffuse intrinsic pontine glioma. J Neurooncol, 2012. 106(2): p. 399-407.

39. Cohen, K.J., et al., Temozolomide in the treatment of high-grade gliomas in children: a report from the Children's Oncology Group. Neuro Oncol, 2011. 13(3): p. 317-23.

40. Stupp, R., et al., Radiotherapy plus concomitant and adjuvant temozolomide for glioblastoma. N Engl J Med, 2005. 352(10): p. 987-96.

41. Diaz, A.K. and S.J. Baker, The genetic signatures of pediatric high-grade glioma: no longer a one-act play. Semin Radiat Oncol, 2014. 24(4): p. 240-7.

42. Brennan, C.W., et al., The somatic genomic landscape of glioblastoma. Cell, 2013. 155(2): p. 462-77.

43. Cancer Genome Atlas Research, N., Comprehensive genomic characterization defines human glioblastoma genes and core pathways. Nature, 2008. 455(7216): p. 1061-8.

44. Parsons, D.W., et al., An integrated genomic analysis of human glioblastoma multiforme. Science, 2008. 321(5897): p. 1807-12.

45. Sherr, C.J. and F. McCormick, The RB and p53 pathways in cancer. Cancer Cell, 2002. 2(2): p. 103-12.

46. Chalhoub, N. and S.J. Baker, PTEN and the PI3-kinase pathway in cancer. Annu Rev Pathol, 2009. 4: p. 127-50.

47. Ekstrand, A.J., et al., Amplified and rearranged epidermal growth factor receptor genes in human glioblastomas reveal deletions of sequences encoding portions of the N-and/or C-terminal tails. Proc Natl Acad Sci U S A, 1992. 89(10): p. 430913.

48. Humphrey, P.A., et al., Anti-synthetic peptide antibody reacting at the fusion junction of deletion-mutant epidermal growth factor receptors in human glioblastoma. Proc Natl Acad Sci U S A, 1990. 87(11): p. 4207-11.

49. Wong, A.J., et al., Structural alterations of the epidermal growth factor receptor gene in human gliomas. Proc Natl Acad Sci U S A, 1992. 89(7): p. 2965-9.

50. Bax, D.A., et al., A distinct spectrum of copy number aberrations in pediatric high-grade gliomas. Clin Cancer Res, 2010. 16(13): p. 3368-77.

51. Paugh, B.S., et al., Genome-wide analyses identify recurrent amplifications of receptor tyrosine kinases and cell-cycle regulatory genes in diffuse intrinsic pontine glioma. J Clin Oncol, 2011. 29(30): p. 3999-4006.

52. Paugh, B.S., et al., Integrated molecular genetic profiling of pediatric high-grade gliomas reveals key differences with the adult disease. J Clin Oncol, 2010. 28(18): p. 3061-8. 
53. Pollack, I.F., et al., Rarity of PTEN deletions and EGFR amplification in malignant gliomas of childhood: results from the Children's Cancer Group 945 cohort. J Neurosurg, 2006. 105(5 Suppl): p. 418-24.

54. Sung, T., et al., Preferential inactivation of the p53 tumor suppressor pathway and lack of EGFR amplification distinguish de novo high grade pediatric astrocytomas from de novo adult astrocytomas. Brain Pathol, 2000. 10(2): p. 24959.

55. Zarghooni, M., et al., Whole-genome profiling of pediatric diffuse intrinsic pontine gliomas highlights platelet-derived growth factor receptor alpha and poly (ADP-ribose) polymerase as potential therapeutic targets. J Clin Oncol, 2010. 28(8): p. 1337-44.

56. Qu, H.Q., et al., Genome-wide profiling using single-nucleotide polymorphism arrays identifies novel chromosomal imbalances in pediatric glioblastomas. Neuro Oncol, 2010. 12(2): p. 153-63.

57. Gilbertson, R.J., et al., ERBB1 is amplified and overexpressed in high-grade diffusely infiltrative pediatric brain stem glioma. Clin Cancer Res, 2003. 9(10 Pt 1): p. 3620-4.

58. Bax, D.A., et al., EGFRvIII deletion mutations in pediatric high-grade glioma and response to targeted therapy in pediatric glioma cell lines. Clin Cancer Res, 2009. 15(18): p. 5753-61.

59. Li, G., et al., Expression of epidermal growth factor variant III (EGFRvIII) in pediatric diffuse intrinsic pontine gliomas. J Neurooncol, 2012. 108(3): p. 395402.

60. Wong, K.K., et al., Genome-wide allelic imbalance analysis of pediatric gliomas by single nucleotide polymorphic allele array. Cancer Res, 2006. 66(23): p. 11172-8.

61. Puget, S., et al., Mesenchymal transition and PDGFRA amplification/mutation are key distinct oncogenic events in pediatric diffuse intrinsic pontine gliomas. PLoS One, 2012. 7(2): p. e30313.

62. Grill, J., et al., Critical oncogenic mutations in newly diagnosed pediatric diffuse intrinsic pontine glioma. Pediatr Blood Cancer, 2012. 58(4): p. 489-91.

63. Barrow, J., et al., Homozygous loss of ADAM3A revealed by genome-wide analysis of pediatric high-grade glioma and diffuse intrinsic pontine gliomas. Neuro Oncol, 2011. 13(2): p. 212-22.

64. Baruchel, S., et al., A Canadian paediatric brain tumour consortium (CPBTC) phase II molecularly targeted study of imatinib in recurrent and refractory paediatric central nervous system tumours. Eur J Cancer, 2009. 45(13): p. 2352-9.

65. Broniscer, A., et al., Phase I trial, pharmacokinetics, and pharmacodynamics of vandetanib and dasatinib in children with newly diagnosed diffuse intrinsic pontine glioma. Clin Cancer Res, 2013. 19(11): p. 3050-8.

66. Pollack, I.F., et al., Phase I trial of imatinib in children with newly diagnosed brainstem and recurrent malignant gliomas: a Pediatric Brain Tumor Consortium report. Neuro Oncol, 2007. 9(2): p. 145-60.

67. Sturm, D., et al., Paediatric and adult glioblastoma: multiform (epi)genomic culprits emerge. Nat Rev Cancer, 2014. 14(2): p. 92-107. 
68. Gallia, G.L., et al., PIK3CA gene mutations in pediatric and adult glioblastoma multiforme. Mol Cancer Res, 2006. 4(10): p. 709-14.

69. Schwartzentruber, J., et al., Driver mutations in histone H3.3 and chromatin remodelling genes in paediatric glioblastoma. Nature, 2012. 482(7384): p. 22631.

70. Broderick, D.K., et al., Mutations of PIK3CA in anaplastic oligodendrogliomas, high-grade astrocytomas, and medulloblastomas. Cancer Res, 2004. 64(15): p. 5048-50.

71. Hartmann, C., et al., PIK3CA mutations in glioblastoma multiforme. Acta Neuropathol, 2005. 109(6): p. 639-42.

72. Samuels, Y., et al., High frequency of mutations of the PIK3CA gene in human cancers. Science, 2004. 304(5670): p. 554.

73. Kraus, J.A., et al., Molecular genetic analysis of the TP53, PTEN, CDKN2A, EGFR, CDK4 and MDM2 tumour-associated genes in supratentorial primitive neuroectodermal tumours and glioblastomas of childhood. Neuropathol Appl Neurobiol, 2002. 28(4): p. 325-33.

74. Raffel, C., et al., Analysis of oncogene and tumor suppressor gene alterations in pediatric malignant astrocytomas reveals reduced survival for patients with PTEN mutations. Clin Cancer Res, 1999. 5(12): p. 4085-90.

75. Cheng, Y., et al., Genetic alterations in pediatric high-grade astrocytomas. Hum Pathol, 1999. 30(11): p. 1284-90.

76. Warren, K.E., et al., Genomic aberrations in pediatric diffuse intrinsic pontine gliomas. Neuro Oncol, 2012. 14(3): p. 326-32.

77. Barton, K.L., et al., PD-0332991, a CDK4/6 inhibitor, significantly prolongs survival in a genetically engineered mouse model of brainstem glioma. PLoS One, 2013. 8(10): p. e77639.

78. Sure, U., et al., Determination of p53 mutations, EGFR overexpression, and loss of p16 expression in pediatric glioblastomas. J Neuropathol Exp Neurol, 1997. 56(7): p. 782-9.

79. Antonelli, M., et al., Prognostic significance of histological grading, p53 status, YKL-40 expression, and IDH1 mutations in pediatric high-grade gliomas. J Neurooncol, 2010. 99(2): p. 209-15.

80. Phillips, H.S., et al., Molecular subclasses of high-grade glioma predict prognosis, delineate a pattern of disease progression, and resemble stages in neurogenesis. Cancer Cell, 2006. 9(3): p. 157-73.

81. Freije, W.A., et al., Gene expression profiling of gliomas strongly predicts survival. Cancer Res, 2004. 64(18): p. 6503-10.

82. Huse, J.T., H.S. Phillips, and C.W. Brennan, Molecular subclassification of diffuse gliomas: seeing order in the chaos. Glia, 2011. 59(8): p. 1190-9.

83. Northcott, P.A., et al., Medulloblastomics: the end of the beginning. Nat Rev Cancer, 2012. 12(12): p. 818-34.

84. Faury, D., et al., Molecular profiling identifies prognostic subgroups of pediatric glioblastoma and shows increased YB-1 expression in tumors. J Clin Oncol, 2007. 25(10): p. 1196-208. 
85. Haque, T., et al., Gene expression profiling from formalin-fixed paraffinembedded tumors of pediatric glioblastoma. Clin Cancer Res, 2007. 13(21): p. 6284-92.

86. Broniscer, A., et al., Prospective collection of tissue samples at autopsy in children with diffuse intrinsic pontine glioma. Cancer, 2010. 116(19): p. 4632-7.

87. Roujeau, T., et al., Stereotactic biopsy of diffuse pontine lesions in children. J Neurosurg, 2007. 107(1 Suppl): p. 1-4.

88. Wu, G., et al., Somatic histone H3 alterations in pediatric diffuse intrinsic pontine gliomas and non-brainstem glioblastomas. Nat Genet, 2012. 44(3): p. 251-3.

89. Khuong-Quang, D.A., et al., K27M mutation in histone H3.3 defines clinically and biologically distinct subgroups of pediatric diffuse intrinsic pontine gliomas. Acta Neuropathol, 2012. 124(3): p. 439-47.

90. Elsaesser, S.J., A.D. Goldberg, and C.D. Allis, New functions for an old variant: no substitute for histone H3.3. Curr Opin Genet Dev, 2010. 20(2): p. 110-7.

91. Yuen, B.T. and P.S. Knoepfler, Histone H3.3 mutations: a variant path to cancer. Cancer Cell, 2013. 24(5): p. 567-74.

92. Schiffman, J.D., et al., Oncogenic BRAF mutation with CDKN2A inactivation is characteristic of a subset of pediatric malignant astrocytomas. Cancer Res, 2010. 70(2): p. 512-9.

93. Sturm, D., et al., Hotspot mutations in H3F3A and IDH1 define distinct epigenetic and biological subgroups of glioblastoma. Cancer Cell, 2012. 22(4): p. 425-37.

94. Pollack, I.F., et al., Age and TP53 mutation frequency in childhood malignant gliomas: results in a multi-institutional cohort. Cancer Res, 2001. 61(20): p. 7404-7.

95. Shore, E.M. and F.S. Kaplan, Role of altered signal transduction in heterotopic ossification and fibrodysplasia ossificans progressiva. Curr Osteoporos Rep, 2011. 9(2): p. 83-8.

96. Shore, E.M., et al., A recurrent mutation in the BMP type I receptor ACVR1 causes inherited and sporadic fibrodysplasia ossificans progressiva. Nat Genet, 2006. 38(5): p. 525-7.

97. Chaikuad, A., et al., Structure of the bone morphogenetic protein receptor ALK2 and implications for fibrodysplasia ossificans progressiva. J Biol Chem, 2012. 287(44): p. 36990-8.

98. Bagarova, J., et al., Constitutively active ALK2 receptor mutants require type II receptor cooperation. Mol Cell Biol, 2013. 33(12): p. 2413-24.

99. Shen, Q., et al., The fibrodysplasia ossificans progressiva R206H ACVR1 mutation activates BMP-independent chondrogenesis and zebrafish embryo ventralization. J Clin Invest, 2009. 119(11): p. 3462-72.

100. Cannon, J.E., et al., Intersegmental vessel formation in zebrafish: requirement for $V E G F$ but not BMP signalling revealed by selective and non-selective BMP antagonists. Br J Pharmacol, 2010. 161(1): p. 140-9.

101. Bond, A.M., O.G. Bhalala, and J.A. Kessler, The dynamic role of bone morphogenetic proteins in neural stem cell fate and maturation. Dev Neurobiol, 2012. 72(7): p. 1068-84. 
102. Zhao, H., et al., Post-transcriptional down-regulation of Atoh1/Math1 by bone morphogenic proteins suppresses medulloblastoma development. Genes Dev, 2008. 22(6): p. 722-7.

103. Piccirillo, S.G., et al., Bone morphogenetic proteins inhibit the tumorigenic potential of human brain tumour-initiating cells. Nature, 2006. 444(7120): p. 7615 .

104. Lee, J., et al., Epigenetic-mediated dysfunction of the bone morphogenetic protein pathway inhibits differentiation of glioblastoma-initiating cells. Cancer Cell, 2008. 13(1): p. 69-80.

105. Harel, L., B. Costa, and M. Fainzilber, On the death Trk. Dev Neurobiol, 2010. 70(5): p. 298-303.

106. Thiele, C.J., Z. Li, and A.E. McKee, On Trk--the TrkB signal transduction pathway is an increasingly important target in cancer biology. Clin Cancer Res, 2009. 15(19): p. 5962-7.

107. Jones, D.T., et al., Recurrent somatic alterations of FGFR1 and NTRK2 in pilocytic astrocytoma. Nat Genet, 2013. 45(8): p. 927-32.

108. Zhang, J., et al., Whole-genome sequencing identifies genetic alterations in pediatric low-grade gliomas. Nat Genet, 2013. 45(6): p. 602-12.

109. Frattini, V., et al., The integrated landscape of driver genomic alterations in glioblastoma. Nat Genet, 2013. 45(10): p. 1141-9.

110. Butti, M.G., et al., A sequence analysis of the genomic regions involved in the rearrangements between TPM3 and NTRK1 genes producing TRK oncogenes in papillary thyroid carcinomas. Genomics, 1995. 28(1): p. 15-24.

111. Cetinbas, N., et al., Mutation of the salt bridge-forming residues in the ETV6SAM domain interface blocks ETV6-NTRK3-induced cellular transformation. J Biol Chem, 2013. 288(39): p. 27940-50.

112. Eguchi, M., et al., Fusion of ETV6 to neurotrophin-3 receptor TRKC in acute myeloid leukemia with t(12;15)(p13;q25). Blood, 1999. 93(4): p. 1355-63.

113. Lannon, C.L. and P.H. Sorensen, ETV6-NTRK3: a chimeric protein tyrosine kinase with transformation activity in multiple cell lineages. Semin Cancer Biol, 2005. 15(3): p. 215-23.

114. Li, Z., et al., ETV6-NTRK3 fusion oncogene initiates breast cancer from committed mammary progenitors via activation of AP1 complex. Cancer Cell, 2007. 12(6): p. 542-58.

115. Fontebasso, A.M., et al., Mutations in SETD2 and genes affecting histone H3K36 methylation target hemispheric high-grade gliomas. Acta Neuropathol, 2013. 125(5): p. 659-69.

116. Bender, S., et al., Reduced H3K27me3 and DNA hypomethylation are major drivers of gene expression in K27M mutant pediatric high-grade gliomas. Cancer Cell, 2013. 24(5): p. 660-72.

117. Chan, K.M., et al., The histone H3.3K27M mutation in pediatric glioma reprograms H3K27 methylation and gene expression. Genes Dev, 2013. 27(9): p. 985-90.

118. Lewis, P.W., et al., Inhibition of PRC2 activity by a gain-of-function H3 mutation found in pediatric glioblastoma. Science, 2013. 340(6134): p. 857-61. 
119. Venneti, S., et al., Evaluation of histone 3 lysine 27 trimethylation (H3K27me3) and enhancer of Zest 2 (EZH2) in pediatric glial and glioneuronal tumors shows decreased H3K27me3 in H3F3A K27M mutant glioblastomas. Brain Pathol, 2013. 23(5): p. 558-64.

120. Fullgrabe, J., E. Kavanagh, and B. Joseph, Histone onco-modifications. Oncogene, 2011. 30(31): p. 3391-403.

121. Zhang, J., et al., The genetic basis of early T-cell precursor acute lymphoblastic leukaemia. Nature, 2012. 481(7380): p. 157-63.

122. Dubuc, A.M., et al., Aberrant patterns of H3K4 and H3K27 histone lysine methylation occur across subgroups in medulloblastoma. Acta Neuropathol, 2013. 125(3): p. 373-84.

123. Jones, D.T., et al., Dissecting the genomic complexity underlying medulloblastoma. Nature, 2012. 488(7409): p. 100-5.

124. Pugh, T.J., et al., Medulloblastoma exome sequencing uncovers subtype-specific somatic mutations. Nature, 2012. 488(7409): p. 106-10.

125. Robinson, G., et al., Novel mutations target distinct subgroups of medulloblastoma. Nature, 2012. 488(7409): p. 43-8.

126. Lin, C.Y., et al., Transcriptional Amplification in Tumor Cells with Elevated cMyc. Cell, 2012. 151(1): p. 56-67.

127. Nie, Z.Q., et al., c-Myc Is a Universal Amplifier of Expressed Genes in Lymphocytes and Embryonic Stem Cells. Cell, 2012. 151(1): p. 68-79.

128. Stephens, P.J., et al., Massive genomic rearrangement acquired in a single catastrophic event during cancer development. Cell, 2011. 144(1): p. 27-40.

129. Killela, P.J., et al., TERT promoter mutations occur frequently in gliomas and a subset of tumors derived from cells with low rates of self-renewal. Proc Natl Acad Sci U S A, 2013. 110(15): p. 6021-6.

130. Kleiblova, P., et al., Gain-of-function mutations of PPM1D/Wip1 impair the p53dependent G1 checkpoint. J Cell Biol, 2013. 201(4): p. 511-21.

131. Sievert, A.J. and M.J. Fisher, Pediatric low-grade gliomas. J Child Neurol, 2009. 24(11): p. 1397-408.

132. Sanders, R.P., et al., High-grade astrocytoma in very young children. Pediatr Blood Cancer, 2007. 49(7): p. 888-93.

133. Cohen, S. and R. Levi-Montalcini, Purification and properties of a nerve growthpromoting factor isolated from mouse sarcoma 180. Cancer Res, 1957. 17(1): p. $15-20$.

134. Levi-Montalcini, R. and S. Cohen, In Vitro and in Vivo Effects of a Nerve Growth-Stimulating Agent Isolated from Snake Venom. Proc Natl Acad Sci U S A, 1956. 42(9): p. 695-9.

135. Lewin, G.R. and B.D. Carter, Neurotrophic factors. Preface. Handb Exp Pharmacol, 2014. 220: p. v-vi.

136. Seidah, N.G., et al., Cellular processing of the neurotrophin precursors of NT3 and BDNF by the mammalian proprotein convertases. FEBS Lett, 1996. 379(3): p. 247-50.

137. Stoleru, B., et al., Tropomyosin-receptor-kinases signaling in the nervous system. Maedica (Buchar), 2013. 8(1): p. 43-8. 
138. West, A.E., P. Pruunsild, and T. Timmusk, Neurotrophins: transcription and translation. Handb Exp Pharmacol, 2014. 220: p. 67-100.

139. Deinhardt, K. and M.V. Chao, Trk receptors. Handb Exp Pharmacol, 2014. 220 : p. 103-19.

140. Kraemer, B.R., S.O. Yoon, and B.D. Carter, The biological functions and signaling mechanisms of the p75 neurotrophin receptor. Handb Exp Pharmacol, 2014. 220: p. 121-64.

141. Martin-Zanca, D., S.H. Hughes, and M. Barbacid, A human oncogene formed by the fusion of truncated tropomyosin and protein tyrosine kinase sequences. Nature, 1986. 319(6056): p. 743-8.

142. Vaishnavi, A., A.T. Le, and R.C. Doebele, TRKing down an old oncogene in a new era of targeted therapy. Cancer Discov, 2015. 5(1): p. 25-34.

143. Bajenaru, M.L., et al., Astrocyte-specific inactivation of the neurofibromatosis 1 gene (NF1) is insufficient for astrocytoma formation. Mol Cell Biol, 2002. 22(14): p. 5100-13.

144. Jonkers, J., et al., Synergistic tumor suppressor activity of BRCA2 and p53 in a conditional mouse model for breast cancer. Nat Genet, 2001. 29(4): p. 418-25.

145. Tronche, F., et al., Disruption of the glucocorticoid receptor gene in the nervous system results in reduced anxiety. Nat Genet, 1999. 23(1): p. 99-103.

146. Srinivas, S., et al., Cre reporter strains produced by targeted insertion of EYFP and ECFP into the ROSA26 locus. BMC Dev Biol, 2001. 1: p. 4.

147. Knezevich, S.R., et al., A novel ETV6-NTRK3 gene fusion in congenital fibrosarcoma. Nat Genet, 1998. 18(2): p. 184-7.

148. Knezevich, S.R., et al., ETV6-NTRK3 gene fusions and trisomy 11 establish a histogenetic link between mesoblastic nephroma and congenital fibrosarcoma. Cancer Res, 1998. 58(22): p. 5046-8.

149. Rubin, B.P., et al., Congenital mesoblastic nephroma t $12 ; 15)$ is associated with ETV6-NTRK3 gene fusion: cytogenetic and molecular relationship to congenital (infantile) fibrosarcoma. Am J Pathol, 1998. 153(5): p. 1451-8.

150. Rahman, M.S., et al., TGF-beta/BMP signaling and other molecular events: regulation of osteoblastogenesis and bone formation. Bone Res, 2015. 3: p. 15005.

151. Hino, K., et al., Neofunction of ACVRl in fibrodysplasia ossificans progressiva. Proc Natl Acad Sci U S A, 2015. 112(50): p. 15438-43.

152. Hatsell, S.J., et al., ACVR1R206H receptor mutation causes fibrodysplasia ossificans progressiva by imparting responsiveness to activin A. Sci Transl Med, 2015. 7(303): p. 303ra137.

153. Gomes, W.A., M.F. Mehler, and J.A. Kessler, Transgenic overexpression of BMP4 increases astroglial and decreases oligodendroglial lineage commitment. Dev Biol, 2003. 255(1): p. 164-77.

154. Monje, M., et al., Hedgehog-responsive candidate cell of origin for diffuse intrinsic pontine glioma. Proc Natl Acad Sci U S A, 2011. 108(11): p. 4453-8.

155. Wai, D.H., et al., The ETV6-NTRK3 gene fusion encodes a chimeric protein tyrosine kinase that transforms NIH3T3 cells. Oncogene, 2000. 19(7): p. 906-15.

156. Mochly-Rosen, D., K. Das, and K.V. Grimes, Protein kinase C, an elusive therapeutic target? Nat Rev Drug Discov, 2012. 11(12): p. 937-57. 
157. de Boer, J., V. Walf-Vorderwulbecke, and O. Williams, In focus: MLLrearranged leukemia. Leukemia, 2013. 27(6): p. 1224-8.

158. Baker, S.J., D.W. Ellison, and D.H. Gutmann, Pediatric gliomas as neurodevelopmental disorders. Glia, 2015.

159. Liu, C., et al., Mosaic analysis with double markers reveals tumor cell of origin in glioma. Cell, 2011. 146(2): p. 209-21.

160. Hanahan, D. and R.A. Weinberg, Hallmarks of cancer: the next generation. Cell, 2011. 144(5): p. 646-74.

161. Hanahan, D. and R.A. Weinberg, The hallmarks of cancer. Cell, 2000. 100(1): p. 57-70. 


\section{VITA}

Alexander K. Diaz was born in 1984. He attended the University of Georgia and graduated with a Bachelor of Science in Psychology in 2007. In 2009, he matriculated at the George Washington University School of Medicine. After completing his first two years of training, Alexander took academic leave and enrolled in the Integrated Program in Biomedical Sciences at the University of Tennessee Health Science Center. He joined the lab of Dr. Suzanne J. Baker in the Department of Developmental Neurobiology at St. Jude Children's Research Hospital where he has studied the genetic and molecular underpinnings of a particularly aggressive brain tumor known as high-grade glioma. He is expected to graduate in May 2016 with a Doctor of Philosophy and will then join the University of Tennessee medical school class of 2018. 